\title{
Short-range three-nucleon interaction from $A=3$ data and its hierarchical structure
}

\author{
L. Girlanda, ${ }^{1,2}$ A. Kievsky, ${ }^{3}$ M. Viviani, ${ }^{3}$ and L. E. Marcucci ${ }^{3,4}$ \\ ${ }^{1}$ Dipartimento di Matematica e Fisica “E. De Giorgi”, Università del Salento, I-73100 Lecce, Italy \\ ${ }^{2}$ INFN, Sezione di Lecce, I-73100 Lecce, Italy \\ ${ }^{3}$ INFN, Sezione di Pisa, I-56127 Pisa, Italy \\ ${ }^{4}$ Dipartimento di Fisica “E. Fermi”, Università di Pisa, I-56127 Pisa, Italy
}

(Received 23 November 2018; revised manuscript received 27 March 2019; published 21 May 2019)

\begin{abstract}
We construct accurate models of the three-nucleon $(3 N)$ interaction by fitting, in a hybrid phenomenological approach, the low-energy constants parametrizing the subleading $3 N$ contact operators to the triton binding energy, $n-d$ scattering lengths, cross section, and polarization observables of $p$ - $d$ scattering at $2 \mathrm{MeV}$ center-ofmass energy. These models lead to a satisfactory description of polarized $p$ - $d$ scattering data in the whole energy range below the deuteron breakup threshold. In particular, the longstanding $A_{y}$ puzzle seems to be solved thanks to the new terms considered in the $3 N$ force. Two types of hierarchies among the subleading contact operators are also derived, based on the large- $N_{c}$ counting and on a recently proposed relativistic counting. We test these hierarchies against the same experimental data and show that they are respected at a reasonable level.
\end{abstract}

DOI: 10.1103/PhysRevC.99.054003

\section{INTRODUCTION}

Recent years have witnessed substantial progress in the development of accurate representations of the nuclear interaction, in both the two-nucleon $(N N)$ and three-nucleon $(3 N)$ sectors [1-8]. Particular emphasis has been put on the systematic framework provided by chiral effective field theory (ChEFT) $[9,10]$. The utility of chiral symmetry as organizing principle of the various components of the nuclear interaction depends on the convergence properties of the corresponding perturbation series, which reflect in turn the separation of the scales at which the nuclear interaction reveals its full complexity. In the $N N$ sector, chiral potentials, developed up to the fourth and fifth order of the low-energy expansion, provide an extremely accurate description of the $N N$ data up to laboratory energies of $300 \mathrm{MeV}$ with a $\chi^{2}$ per degree of freedom ( $\chi^{2} /$ d.o.f.) close to one. The three-nucleon interaction (TNI) shows up in this framework as a small perturbation to the $N N$ interaction arising at the next-to-next-to-leading order (N2LO), and depends only on two low-energy constants (LECs) up to following order N3LO [11,12]. After determining the two TNI LECs from two $3 N$ data (usually they are the ${ }^{3} \mathrm{H}$ binding energy and the doublet $n$ - $d$ scattering length or tritium $\beta$ decay) the calculated $\chi^{2} /$ d.o.f. of available low-energy $N$ - $d$ observables takes values as large as several hundreds $[13,14]$. This well-known fact regards unexplained discrepancies between theory and experiment in low-energy $N-d$ scattering, most notably in polarization observables of elastic scattering, as the so-called $A_{y}$ puzzle [15-17]. Attempts to trace back this problem to deficiencies in the description of the low-energies $N N p$ waves showed that it is impossible to simultaneously describe the low-energy $N N$ and $3 N$ database using solely $N N$ forces [18]. Accordingly, these discrepancies indicate a limited flexibility in the $3 N$ force at the order considered. To improve the description, further LECs, parametrizing subleading contact terms contributing at N4LO, could be necessary. This would imply a slower convergence of the ChEFT series than expected, or the necessity of promoting short-range contact terms in the low-energy counting [19-22]. In the present paper we focus on this component of the TNI to assess its relevance in the resolution of the above discrepancies. The subleading TNI contact potential has been derived in Ref. [23]. It was shown that it consists of ten independent terms involving different combinations of the space-spin-isospin variables. Preliminary studies [24] already indicated that the associated operatorial structures provide enough flexibility to improve the description of polarization observables in low-energy $\mathrm{N}-d$ scattering. In particular, assigning values to some of the accompanying LECs, it is possible to describe the two vector analyzing powers $A_{y}$ and $i T_{11}$ in good agreement with the experimental data. This preliminary study has opened the door to the possibility of fixing the TNI LECs from $3 N$ scattering data. In the present paper we intend to start a systematic study using $N-d$ scattering data to fix the ten contact TNI LECs from a fitting procedure similar to what is done in the determination of the $N N$ interaction. As a first step in this direction, and following the previous analysis, we take the leading part of the force to be the $\operatorname{AV} 18 N N$ potential [25], with only the point-Coulomb interaction retained in the electromagnetic terms, in conjunction with the Urbana IX (UIX) model of TNI [26]. We fit the corresponding LECs to very precise $p$ - $d$ cross section and polarization observables at center of mass energy $E_{\mathrm{cm}}=2 \mathrm{MeV}$ (or proton energy $E_{p}=3 \mathrm{MeV}$ ) [27] for different choices of the contact shortdistance cutoff $\Lambda$ between 200 and $500 \mathrm{MeV}$. The resulting Hamiltonian is then used to predict the observables at other energies with an overall satisfactory agreement inside the energy range explored.

On a more formal ground, we derive a hierarchy among these LECs as dictated by 't Hooft large- $N_{c}$ limit of QCD 
$[28,29]$. We also consider a recently proposed alternative counting for contact operators, which does not rely on the nonrelativistic expansion for nucleons [30], and classify the $3 N$ contact operators appearing at the leading order in this counting. The simplified models for the contact TNI resulting from the leading orders of these schemes are also tested against the same experimental data, obtaining results of comparable quality. In particular, the relativistic counting seems to provide a natural explanation for a large spin-orbit term, as requested to explain the $A_{y}$ puzzle [31]. Strictly speaking these expansion schemes could only be tested in association with a chiral $N N$ potential derived in the same framework. However, we take the indications from the present hybrid approach as suggestive of their effectiveness.

The paper is organized as follows. In Sec. II we present our model of TNI interaction. Since the $p$ - $d$ scattering can mostly probe the isospin $T=1 / 2$ component of the TNI we also discuss the projection of the model in this channel. There is also a $T=3 / 2$ component, which we leave undetermined: it could be fixed by other experimental observables. In Sec. III we describe the variational procedure we use to solve the $p$ - $d$ scattering problem, which is based on the expansion on the hyperspherical harmonics (HH method), and we describe the adopted fitting strategy and the results. The predictions at lower energies are compared to available experimental data in Sec. IV. In Sec. V we determine the simplification of the subleading TNI implied by the large- $N_{c}$ limit and by the relativistic counting, and the corresponding test against experimental data. Finally, Sec. VI contains some concluding remarks. Details of the Fierz identities for covariant nucleon trilinears are collected in the Appendix.

\section{SUBLEADING TNI}

In Ref. [23] all subleading $3 N$ contact terms, compatible with the discrete symmetries of QCD and with the relativity constraints [32], were classified. The Pauli principle severely reduces their number to only ten independent structures. From the Lagrangian density,

$$
\mathcal{L}_{3 N}=-\sum_{i=1}^{10} E_{i} O_{i}
$$

by appropriately choosing the momentum cutoff as dependent only on momentum transfers, an explicit representation of the associated $3 N$ potential can be derived, which is local in coordinate space and depends on a short-distance cutoff $\Lambda$ and the ten subleading LECs $E_{i}, i=1, \ldots, 10$. It is explicitly written as

$$
\begin{aligned}
V^{(2)}= & \sum_{i \neq j \neq k}\left(E_{1}+E_{2} \boldsymbol{\tau}_{i} \cdot \boldsymbol{\tau}_{j}+E_{3} \boldsymbol{\sigma}_{i} \cdot \boldsymbol{\sigma}_{j}+E_{4} \boldsymbol{\tau}_{i} \cdot \boldsymbol{\tau}_{j} \boldsymbol{\sigma}_{i} \cdot \boldsymbol{\sigma}_{j}\right) \\
& \times\left[Z_{0}^{\prime \prime}\left(r_{i j}\right)+2 \frac{Z_{0}^{\prime}\left(r_{i j}\right)}{r_{i j}}\right] Z_{0}\left(r_{i k}\right) \\
& +\left(E_{5}+E_{6} \boldsymbol{\tau}_{i} \cdot \boldsymbol{\tau}_{j}\right) S_{i j}\left[Z_{0}^{\prime \prime}\left(r_{i j}\right)-\frac{Z_{0}^{\prime}\left(r_{i j}\right)}{r_{i j}}\right] Z_{0}\left(r_{i k}\right) \\
& +\left(E_{7}+E_{8} \boldsymbol{\tau}_{i} \cdot \boldsymbol{\tau}_{k}\right)(\mathbf{L} \cdot \boldsymbol{S})_{i j} \frac{Z_{0}^{\prime}\left(r_{i j}\right)}{r_{i j}} Z_{0}\left(r_{i k}\right) \\
& +\left(E_{9}+E_{10} \boldsymbol{\tau}_{j} \cdot \boldsymbol{\tau}_{k}\right) \boldsymbol{\sigma}_{j} \cdot \hat{\mathbf{r}}_{i j} \boldsymbol{\sigma}_{k} \cdot \hat{\mathbf{r}}_{i k} Z_{0}^{\prime}\left(r_{i j}\right) Z_{0}^{\prime}\left(r_{i k}\right),
\end{aligned}
$$

where $S_{i j}$ and $(\mathbf{L} \cdot S)_{i j}$ are, respectively, the tensor and spinorbit operators for particles $i$ and $j$, and the function $Z_{0}(r)$ is the Fourier transform of the cutoff function $F\left(\mathbf{p}^{2} ; \Lambda\right)$,

$$
Z_{0}(r ; \Lambda)=\int \frac{d \mathbf{p}}{(2 \pi)^{3}} e^{i \mathbf{p} \cdot \mathbf{r}} F\left(\mathbf{p}^{2} ; \Lambda\right) .
$$

We adopt the following choice for the cutoff function

$$
F\left(\mathbf{p}^{2} ; \Lambda\right)=\exp \left[-\left(\frac{\mathbf{p}^{2}}{\Lambda^{2}}\right)^{2}\right]
$$

which has the advantage of preserving the low-energy counting up to the order we are considering.

In this paper we consider a nuclear interaction consisting of the AV18 NN potential the UIX TNI and an additional interaction given by

$$
V_{3 N}^{C T}=V^{(0)}+V^{(2)}
$$

where the leading $3 N$ contact potential $V^{(0)}$ is written as

$$
V^{(0)}=\sum_{i \neq j \neq k} E_{0} Z_{0}\left(r_{i j}\right) Z_{0}\left(r_{i k}\right)
$$

Since the deuteron is an isosinglet state, matrix elements between $N-d$ states only probe the total $T=1 / 2$ component of the TNI. In order to identify this component we use the projectors on the two isospin channels, which for the threenucleon system take the form

$$
P_{1 / 2}=\frac{1}{2}-\frac{1}{6}\left(\boldsymbol{\tau}_{1} \cdot \boldsymbol{\tau}_{2}+\boldsymbol{\tau}_{1} \cdot \boldsymbol{\tau}_{3}+\boldsymbol{\tau}_{2} \cdot \boldsymbol{\tau}_{3}\right),
$$

and $P_{3 / 2}=1-P_{1 / 2}$. The $3 N$ potential $V^{(2)}$ can be expressed in momentum space as

$$
V^{(2)}=\sum_{i} E_{i} O_{i},
$$

where the ten $O_{i}$ operators are

$$
\begin{aligned}
& O_{1}=-\mathbf{k}_{i}^{2}, \\
& O_{3}=-\mathbf{k}_{i}^{2} \boldsymbol{\sigma}_{i} \cdot \boldsymbol{\sigma}_{j}, \\
& O_{5}=-3 \mathbf{k}_{i} \cdot \boldsymbol{\sigma}_{i} \mathbf{k}_{i} \cdot \boldsymbol{\sigma}_{j}+\mathbf{k}_{i}^{2} \boldsymbol{\sigma}_{i} \cdot \boldsymbol{\sigma}_{j}, \\
& O_{7}=-\frac{i}{4} \mathbf{k}_{i} \times\left(\mathbf{Q}_{i}-\mathbf{Q}_{j}\right) \cdot\left(\boldsymbol{\sigma}_{i}+\boldsymbol{\sigma}_{j}\right), \\
& O_{9}=-\mathbf{k}_{i} \cdot \boldsymbol{\sigma}_{i} \mathbf{k}_{j} \cdot \boldsymbol{\sigma}_{j},
\end{aligned}
$$

$$
\begin{aligned}
& O_{2}=-\mathbf{k}_{i}^{2} \boldsymbol{\tau}_{i} \cdot \boldsymbol{\tau}_{j}, \\
& O_{4}=-\mathbf{k}_{i}^{2} \boldsymbol{\sigma}_{i} \cdot \boldsymbol{\sigma}_{j} \boldsymbol{\tau}_{i} \cdot \boldsymbol{\tau}_{j}, \\
& O_{6}=\left(-3 \mathbf{k}_{i} \cdot \boldsymbol{\sigma}_{i} \mathbf{k}_{i} \cdot \boldsymbol{\sigma}_{j}+\mathbf{k}_{i}^{2} \boldsymbol{\sigma}_{i} \cdot \boldsymbol{\sigma}_{j},\right) \boldsymbol{\tau}_{i} \cdot \boldsymbol{\tau}_{j}, \\
& O_{8}=-\frac{i}{4} \mathbf{k}_{i} \times\left(\mathbf{Q}_{i}-\mathbf{Q}_{j}\right) \cdot\left(\boldsymbol{\sigma}_{i}+\boldsymbol{\sigma}_{j}\right) \boldsymbol{\tau}_{j} \cdot \boldsymbol{\tau}_{k}, \\
& O_{10}=-\mathbf{k}_{i} \cdot \boldsymbol{\sigma}_{i} \mathbf{k}_{j} \cdot \boldsymbol{\sigma}_{j} \boldsymbol{\tau}_{i} \cdot \boldsymbol{\tau}_{j},
\end{aligned}
$$


with $\mathbf{k}_{i}=\mathbf{p}_{i}-\mathbf{p}_{i}^{\prime}, \mathbf{Q}_{i}=\mathbf{p}_{i}+\mathbf{p}_{i}^{\prime}$, and $\mathbf{p}_{i}\left(\mathbf{p}_{i}^{\prime}\right)$ the initial (final) momentum of the $i$ th nucleon, and a sum over $i \neq j \neq k$ is understood. The projections over isospin $T=1 / 2,\left(O_{i}\right)_{1 / 2}=$ $P_{1 / 2} O_{i} P_{1 / 2}$ are given, using the relations derived in Ref. [23], by

$$
\begin{aligned}
\left(O_{1}\right)_{1 / 2}= & O_{1}-\frac{1}{3} O_{2}+\frac{1}{3} O_{3}+\frac{1}{9} O_{4}+\frac{1}{3} O_{5} \\
& +\frac{1}{9} O_{6}+4 O_{7}+\frac{4}{3} O_{8}+O_{9}+\frac{1}{3} O_{10} \\
\left(O_{2}\right)_{1 / 2}= & \frac{2}{3} O_{2}+\frac{1}{3} O_{3}+\frac{1}{9} O_{4}+\frac{1}{3} O_{5} \\
& +\frac{1}{9} O_{6}+4 O_{7}+\frac{4}{3} O_{8}+O_{9}+\frac{1}{3} O_{10} \\
\left(O_{i}\right)_{1 / 2}= & O_{i}, \quad i=3, \ldots, 8 \\
\left(O_{9}\right)_{1 / 2}= & \frac{1}{6} O_{2}-\frac{1}{6} O_{3}-\frac{1}{18} O_{4}-\frac{1}{6} O_{5} \\
& -\frac{1}{18} O_{6}-2 O_{7}-\frac{2}{3} O_{8}+\frac{1}{2} O_{9}-\frac{1}{6} O_{10}, \\
\left(O_{10}\right)_{1 / 2}= & \frac{1}{6} O_{2}-\frac{1}{6} O_{3}-\frac{1}{18} O_{4}-\frac{1}{6} O_{5} \\
& -\frac{1}{18} O_{6}-2 O_{7}-\frac{2}{3} O_{8}-\frac{1}{2} O_{9}+\frac{5}{6} O_{10}
\end{aligned}
$$

By examining the above relations, we find that there are nine purely $T=1 / 2$ combinations, e.g.,

$$
\left(O_{1}-O_{2}\right), \quad\left(O_{2}+2 O_{10}\right), \quad O_{i=3, \ldots, 8}, \quad\left(O_{9}-O_{10}\right),
$$

and a single purely $T=3 / 2$ combination of operators, e.g.,

$$
\begin{aligned}
O_{3 / 2}= & 3 O_{2}-3 O_{3}-O_{4}-3 O_{5}-O_{6}-36 O_{7} \\
& -12 O_{8}-9 O_{9}-3 O_{10} .
\end{aligned}
$$

Notice that, in order to derive the above projections, Fierz transformations have been repeatedly used. Therefore the conclusion only holds up to cutoff effects: indeed the cutoff smears the contact interactions and, as a consequence, the three nucleons, no longer at the same position, are much less constrained by the Pauli principle. Thus, only nine combinations of LECs may enter $p$ - $d$ observables, and no full determination of all the ten LECs will be possible without adding an extra $T=3 / 2$ observable. We may as well start from a Hamiltonian written in terms of the isospin-projected operators Eqs. (15) and (16) with LECs $h_{i}, i=1, \ldots, 9$, and $h_{3 / 2}$, respectively, in one-to-one correspondence with the $E_{i}$, e.g.,

$$
h_{3 / 2}=\frac{1}{18}\left[2\left(E_{1}+E_{2}\right)-E_{9}-E_{10}\right] .
$$

Dropping the $T=3 / 2$ operator from the Hamiltonian, which does not affect the $p$ - $d$ observables, amounts to setting $h_{3 / 2}=0$, leading to the relation

$$
2\left(E_{1}+E_{2}\right)-E_{9}-E_{10}=0 .
$$

Thus we may effectively impose the above constraint when fitting to $p$ - $d$ observables, and shifting all the LECs by an amount proportional to the $T=3 / 2$ LEC multiplying $O_{3 / 2}$ according to Eq. (16), once we add this extra observable.

\section{NUMERICAL DETERMINATION OF THE CONTACT LECS}

We use the HH method to solve the three-body Schrödinger equation, as reviewed in Ref. [33]. The $N-d$ scattering wave function, below the deuteron breakup threshold, is written as the sum of an internal and an asymptotic part,

$$
\Psi_{L S J J_{z}}=\Psi_{\mathrm{C}}+\Psi_{\mathrm{A}},
$$

where the internal part is expanded in hyperspherical harmonics,

$$
\Psi_{C}=\sum_{\mu} c_{\mu} \Phi_{\mu}
$$

$\mu$ denoting a set of quantum numbers necessary to completely specify the basis element, while the asymptotic part, $\Psi_{\mathrm{A}}$, describes the relative motion between the nucleon and the deuteron at large separation, which takes the form of a linear combination of the regular and irregular solutions of the free (or Coulomb) $N-d$ Schrödinger equation at relative momentum $q$ (corresponding to energy $E$ ), duly regulated at small distance. Therefore, denoting these solutions with $\Omega_{L S J J_{z}}^{\lambda}, \lambda=R, I$, respectively, we can write,

$$
\Psi_{A}^{L S J J_{z}}=\Omega_{L S J J_{z}}^{R}+\sum_{L^{\prime} S^{\prime}} \mathcal{R}_{L S, L^{\prime} S^{\prime}}^{J}(q) \Omega_{L^{\prime} S^{\prime} J J_{z}}^{I} .
$$

The weights $\mathcal{R}_{L S, L^{\prime} S^{\prime}}^{J}$ of the irregular solution relative to the regular one are the $K$-matrix elements. It is related to the $S$ matrix from the relation $S=(1+i K)(1-i K)^{-1}$. The $K$ matrix, that determines the scattering phase shifts and mixing parameters, together with the coefficient $c_{\mu}$ in Eq. (20) are obtained from the Kohn variational principle. The principle can be formulated in its real or complex form [34] and requires that the functional

$$
\left[\mathcal{R}_{L S, L^{\prime} S^{\prime}}^{J}(q)\right]=\mathcal{R}_{L S, L^{\prime} S^{\prime}}^{J}(q)-\left\langle\Psi_{L^{\prime} S^{\prime} J J_{z}}|H-E| \Psi_{L S J J_{z}}\right\rangle,
$$

be stationary under changes of the variational parameters in $\Psi_{L S J J_{z}}$, with the asymptotic part normalized such that

$$
\left\langle\Omega_{L S J J_{z}}^{R}|H-E| \Omega_{L S J_{z}}^{I}\right\rangle-\left\langle\Omega_{L S J J_{z}}^{I}|H-E| \Omega_{L S J J_{z}}^{R}\right\rangle=1 .
$$

This implies that the weights $\mathcal{R}_{L S, L^{\prime} S^{\prime}}^{J}$ must solve the linear system

$$
\sum_{\tilde{L} \tilde{S}} \mathcal{R}_{L S, \tilde{L} \tilde{S}}^{J} X_{L^{\prime} S^{\prime}, \tilde{L} \tilde{S}}=Y_{L S, L^{\prime} S^{\prime}}
$$

where

$$
\begin{aligned}
& X_{L S, L^{\prime} S^{\prime}}=\left\langle\Omega_{L S J J_{z}}^{I}+\Psi_{C}^{I}|H-E| \Omega_{L^{\prime} S^{\prime} J J_{z}}^{I}\right\rangle, \\
& Y_{L S, L^{\prime} S^{\prime}}=-\left\langle\Omega_{L S J J_{z}}^{I}+\Psi_{C}^{R}|H-E| \Omega_{L^{\prime} S^{\prime} J J_{z}}^{I}\right\rangle,
\end{aligned}
$$

and the internal functions $\Psi_{C}^{\lambda}$ have coefficients $c_{\mu}^{\lambda}$ solutions of

$$
\sum_{\mu^{\prime}}\left\langle\Phi_{\mu}|H-E| \Phi_{\mu^{\prime}}\right\rangle c_{\mu^{\prime}}^{\lambda}=-\left\langle\Phi_{\mu}|H-E| \Omega_{L S J J_{z}}^{\lambda}\right\rangle
$$

with $\lambda=R, I$. A second-order estimate is then obtained by substituting the obtained weights $\mathcal{R}_{L S, L^{\prime} S^{\prime}}^{J}$ into Eq. (22). From Eqs. (25) and (26) we notice that, in order to solve the linear problem, the matrix elements of the Hamiltonian $H$ have to be computed between the $\mathrm{HH}$ basis elements and the asymptotic functions. Decomposing the Hamiltonian as

$$
H=T+V=T+V_{2 N}+V_{3 N}=H_{L}+V^{(0)}+V^{(2)},
$$


where $H_{L}$ is the leading Hamiltonian containing the kinetic energy $T$ plus the selected two- and three-body force and $V^{(0)}+V^{(2)}$ are the leading and subleading contact interactions, the linear system of Eq. (26) results

$$
\begin{aligned}
& \sum_{\mu^{\prime}} c_{\mu}^{\lambda}\left\langle\Phi_{\mu}\left|H_{L}+\sum_{i=0,10} E_{i} V_{i}-E\right| \Phi_{\mu^{\prime}}\right\rangle \\
& =-\left\langle\Phi_{\mu}\left|H_{L}+\sum_{i=0,10} E_{i} V_{i}-E\right| \Omega_{L S J J_{z}}^{\lambda}\right\rangle
\end{aligned}
$$

which can be put in the matricial form

$$
\begin{gathered}
\sum_{\mu^{\prime}}\left[\left(H_{L}\right)_{\mu \mu^{\prime}}+\sum_{i=0,10} E_{i}\left(V_{i}\right)_{\mu \mu^{\prime}}-E N_{\mu \mu^{\prime}}\right] c_{\mu^{\prime}}^{\lambda} \\
=-\left(H_{L}\right)_{\mu \lambda}+\sum_{i=0,10} E_{i}\left(V_{i}\right)_{\mu \lambda}-E N_{\mu \lambda},
\end{gathered}
$$

where $\left(H_{L}\right)_{\mu \mu^{\prime}}$ denote the matrix elements of $H_{L}$ between the corresponding basis states and similarly for the other operators. Here $E_{i}, V_{i}$ are the contact leading $(i=0)$ and subleading $(i=1, \ldots, 10)$ LECs and operators, respectively. As can be seen the problem has been reduced to a linear one: the contact potential energy can be computed as a linear combination of several matrices, one for $V^{(0)}$ and one for each operator appearing in $V^{(2)}$. These matrices can be computed once for all, weighted by the corresponding LECs. Using the Kohn variational principle in the complex formalism, a particular set of LECs can be used to compute the corresponding $S$ or $T$ matrix for each $J^{\pi}$ state from which the observables at a particular energy $E$ can be obtained. To this end we calculate the $N-d$ transition matrix $M$ decomposed as a sum of the Coulomb amplitude $f_{c}$ plus a nuclear term

$$
\begin{aligned}
M_{v v^{\prime}}^{S S^{\prime}}(\theta)= & f_{c}(\theta) \delta_{S S^{\prime}} \delta_{v v^{\prime}} \\
& +\frac{\sqrt{4 \pi}}{k} \sum_{L, L^{\prime}, J} \sqrt{2 L+1}(L 0 S v \mid J v)\left(L^{\prime} M^{\prime} S^{\prime} v^{\prime} \mid J v\right) \\
& \times \exp \left[i\left(\sigma_{L}+\sigma_{L^{\prime}}-2 \sigma_{0}\right)\right] T_{L S, L^{\prime} S^{\prime}}^{J} Y_{L^{\prime} M^{\prime}}(\theta, 0),
\end{aligned}
$$

where the matrix $M$ is a $6 \times 6$ matrix corresponding to the couplings of the spin 1 and spin $1 / 2$, of the deuteron and third particle, to $S, S^{\prime}=1 / 2$ or $3 / 2$ with projections $v$ and $\nu^{\prime}$. The quantum numbers $L, L^{\prime}$ are the relative orbital angular momentum between the deuteron and the third particle and $J$ is the total angular momentum of the three-nucleon state. The matrix elements $T_{L S, L^{\prime} S^{\prime}}^{J}$ form the $T$ matrix of a Hamiltonian containing the nuclear plus Coulomb interactions, $\sigma_{L}$ are the Coulomb phase shifts. The $n$ - $d$ case is recovered with $f_{c}=$ $\sigma_{L}=0$.

Let us first determine the expected sizes of the LECs, which, according to naive dimensional analysis $[35,36]$, are as follows:

$$
E_{0} \sim \frac{1}{F_{\pi}^{4} \Lambda}, \quad E_{i} \sim \frac{1}{F_{\pi}^{4} \Lambda^{3}}, \quad i=1, \ldots, 10,
$$

where $F_{\pi}=92.4 \mathrm{MeV}$ is the pion decay constant and $\Lambda$ is the hadronic scale. This counting is expected in the pionful theory. In the pionless case the LECs may also receive contributions from virtual pion exchanges, which will produce extra factors of $\Lambda^{2} / M_{\pi}^{2}$. We therefore extract physical dimensions and write

$$
E_{0}=\frac{e_{0}}{F_{\pi}^{4} \Lambda}, \quad E_{i}=\frac{e_{i}}{F_{\pi}^{4} \Lambda^{3}}, \quad i=1, \ldots, 10,
$$

with $e_{0} \sim e_{i} \sim O(1)$ if natural.

In the determination of the LECs we make use of the following data: the triton binding energy, the doublet and quartet $n-d$ scattering lengths $[37,38]$ and several $p$ - $d$ scattering observables at $3 \mathrm{MeV}$ proton energy for which a very precise set of data exists [27]. The $p$ - $d$ observables used for the fit are the differential cross section, the two vector analyzing powers $A_{y}$ and $i T_{11}$ and the three tensor analyzing powers $T_{20}, T_{21}$, and $T_{22}$. For each choice of the ten subleading LECs, subjected to the $T=1 / 2$ constraint (18), we redetermine the leading contact LEC $E_{0}$ from the experimental triton binding energy. We then fit the experimental doublet and quartet $N-d$ scattering length $[37,38]$ and the six $p$ - $d$ scattering observables at $E_{p}=3 \mathrm{MeV}$ [27], amounting to $\sim 300$ experimental data. The theoretical observables are calculated solving Eq. (29) for a set of $E_{i}$ coefficients for different $J^{\pi}$ states. The obtained $S$ matrix (or $T$ matrix) is used to calculate the transition matrix $M$ from which the observables are directly calculated [39]. At the energy considered, states up to $L=2$ are calculated using the full Hamiltonian whereas for $L>2$ only the two-body potential was included up to a maximum value of $L=6$.

For the differential cross section we include in the $\chi^{2}$ an overall normalization factor $Z$ of the data points,

$$
\chi^{2}=\sum_{i} \frac{\left(d_{i}^{\exp } / Z-d_{i}^{\mathrm{th}}\right)^{2}}{\left(\sigma_{i}^{\exp } / Z\right)^{2}},
$$

with $Z$ obtained from the minimization condition as

$$
Z=\frac{\sum_{i} d_{i}^{\exp } d_{i}^{\text {th }} /\left(\sigma_{i}^{\exp }\right)^{2}}{\sum_{i}\left(d_{i}^{\text {th }}\right)^{2} /\left(\sigma_{i}^{\exp }\right)^{2}}
$$

and checked that $Z$ never differs from 1 by more than $2 \%$ [40]. For the other observables, we treat the normalization $Z=1.00 \pm 0.01$ as an experimental datum, to be added to the $\chi^{2}$, since, according to Ref. [27] the systematic uncertainty is estimated as $1 \%$.

For a given initial set of LEC values we use Eq. (29) to solve the scattering problem and calculate the corresponding observables. Using the POUNDerS algorithm [41] we start an iterative procedure to minimize the global $\chi^{2} /$ d.o.f. of the data set description. After several iterations the numerical procedure converges to a local minimum. We repeat the procedure using different initial input of values trying to localize the deepest minimum.

Among the ten contact LECs the most relevant one for all adopted values of the short-distance cutoff $\Lambda$ is the spinorbit one $E_{7}$. One-parameter fits to the considered scattering observables lead to a $\chi^{2} /$ d.o.f. $\sim 4$. The most drastic improvement is obtained fitting two parameters [42]. In particular the least $\chi^{2}$ are reached including the tensor operator parametrized by the LEC $E_{5}$ together with the spin-orbit one, in agreement with previous analyses [31,43]. As shown in Table I, the $\chi^{2} /$ d.o.f. can be reduced to $\sim 2$. The mean values of the different Hamiltonian components inside the triton state 
TABLE I. Two-parameter fit results neglecting all the subleading contact terms except the tensor and spin-orbit operators $O_{5,7}$ on top of the two-body AV18, the three-body UIX potential, and the leading $3 N$ contact potential $V^{(0)}$ given in Eq. (6). The rescaled LEC $e_{0}$ is determined from the triton binding energy, while the doublet and quartet scattering lengths $a_{2}$ and $a_{4}$ are fitted to the experimental values $a_{2}=(0.645 \pm 0.003 \pm 0.007) \mathrm{fm} \mathrm{[37]} \mathrm{and} a_{4}=$ $(6.35 \pm 0.02) \mathrm{fm}[38]$. Also shown are the mean values in the triton state of the one- and two-body Hamiltonian (denoted as $\langle\mathrm{AV} 18\rangle$ ), of the UIX three-body potential $\langle$ UIX $\rangle$, and of individual contributions from the short-distance three-body potential.

\begin{tabular}{lcccc}
\hline \hline$\Lambda(\mathrm{MeV})$ & 200 & 300 & 400 & \multicolumn{1}{c}{500} \\
\hline$\chi^{2} /$ d.o.f. & 2.0 & 2.0 & 2.1 & 2.1 \\
$e_{0}$ & -0.074 & -0.037 & 0.053 & 0.451 \\
$e_{5}$ & -0.212 & -0.248 & -0.403 & -0.799 \\
$e_{7}$ & 1.104 & 1.195 & 1.686 & 2.598 \\
$\langle\mathrm{AV} 18\rangle(\mathrm{MeV})$ & -7.353 & -7.373 & -7.394 & -7.343 \\
$\langle\mathrm{UIX}\rangle(\mathrm{MeV})$ & -1.118 & -1.095 & -1.058 & -1.031 \\
$\left\langle V^{(0)}\right\rangle(\mathrm{MeV})$ & -0.057 & -0.069 & 0.125 & 0.841 \\
$\left\langle E_{5} O_{5}\right\rangle(\mathrm{MeV})$ & -0.032 & -0.182 & -0.609 & -1.553 \\
$\left\langle E_{7} O_{7}\right\rangle(\mathrm{MeV})$ & 0.079 & 0.237 & 0.454 & 0.605 \\
$a_{2}(\mathrm{fm})$ & 0.611 & 0.618 & 0.626 & 0.638 \\
$a_{4}(\mathrm{fm})$ & 6.32 & 6.32 & 6.32 & 6.32 \\
\hline \hline
\end{tabular}

suggest that the included contact operators cease to be a small perturbation to the three-body UIX potential for the largest values of the cutoff $\Lambda$. Also shown in the last two rows of the table are the doublet and quadruplet $n$ - $d$ scattering lengths $a_{2}$ and $a_{4}$ respectively. The fitted curves are shown in Fig. 1 as red narrow bands and a nice description of all the observables is evident. For the sake of comparison we also show the predictions using the two-body interaction AV18 (dashed lines) and the AV18+UIX model (dashed-dotted lines). The underprediction of $A_{y}$ and $i T_{11}$ are well visible in these two cases.

Improvements of the quality of the fit result from inclusion of all the $T=1 / 2$ subleading LECs, as shown in Table II. Values of $\chi^{2} /$ d.o.f. $\lesssim 1.7$, are obtained for all values of the short-distance cutoff between $\Lambda=200$ and $500 \mathrm{MeV}$. The fitted curves are shown in Fig. 2, in good agreement with all observables. We notice that the doublet scattering length and $T_{11}$ observables are better reproduced as compared to the simplified model discussed above. However, the resulting TNI is not a small perturbation, as compared to the UIX. For instance, the contact terms $E_{0}, \ldots, E_{10}$ contribute an overall attraction in the triton binding energy of more than $1 \mathrm{MeV}$, for the largest cutoff, which is also the result of huge cancellations among the different terms. This is also clear from the rather unnatural values of the resulting LECs.

\section{PREDICTIONS AT LOWER ENERGIES}

With the LECs determined at a proton energy of $E_{p}=$ $3 \mathrm{MeV}$, we can predict observables at other energies. To this end we select $p$ - $d$ scattering below the deuteron breakup, and postpone the analysis at energies above the breakup as well as an energy-dependent fit to a forthcoming study. To make

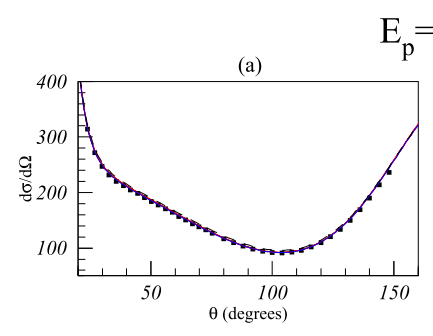

$\mathrm{E}_{\mathrm{p}}=3.0 \mathrm{MeV}$
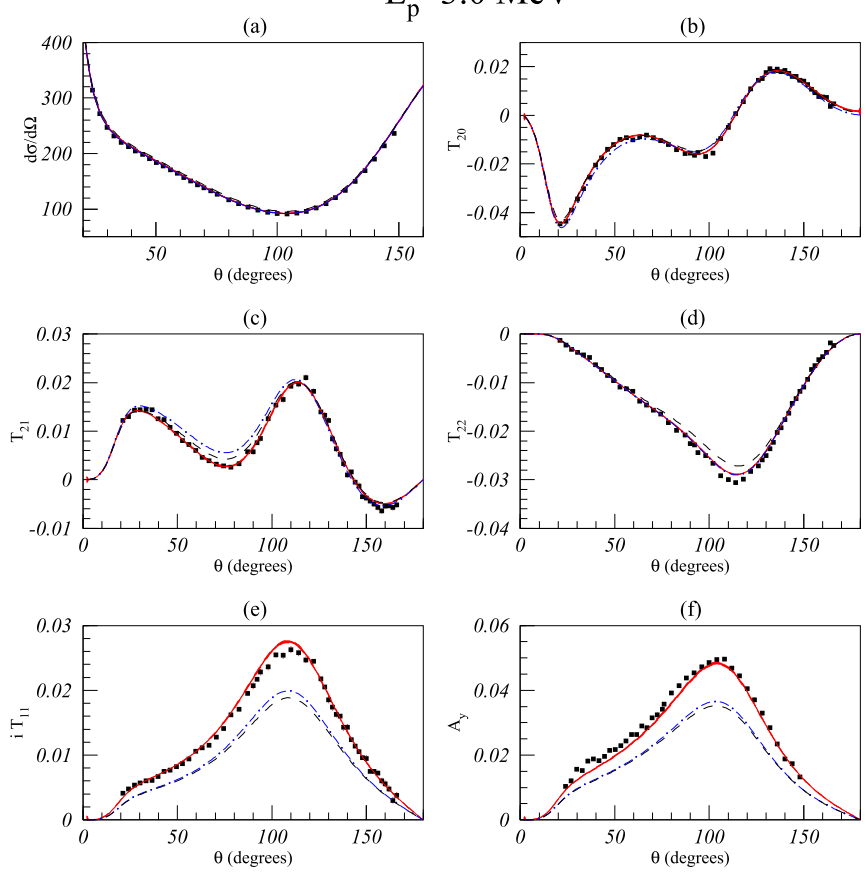

FIG. 1. Fitted curves, including only the tensor and spin-orbit subleading contact operator on the top of the AV18+UIX interaction, to a set of cross-section and polarization observables in $p$ - $d$ scattering at $3 \mathrm{MeV}$ proton energy [27], for $\Lambda=200-500 \mathrm{MeV}$ (red bands) as compared to the purely two-body AV18 interaction (dashed black lines) and to the AV18+UIX two- and three-nucleon interaction (dashed-dotted, blue lines).

this analysis we choose the models corresponding to $\Lambda=$ $300 \mathrm{MeV}$. We have checked the cutoff dependence at proton energy $E_{p}=1.0 \mathrm{MeV}$ and found that it is small between 200 and $500 \mathrm{MeV}$, as shown in Fig. 3.

Several observables have been measured at proton energies of $E_{p}=1.0,2.5,2.0,0.647 \mathrm{MeV}[27,44,45]$. The

TABLE II. Fit results for the models that include all $T=1 / 2$ subleading contact operators in addition to the AV18, the UIX and the leading $3 N$ contact potential for the different cutoff considered.

\begin{tabular}{lcccc}
\hline \hline$\Lambda(\mathrm{MeV})$ & 200 & 300 & 400 & 500 \\
\hline$\chi^{2} /$ d.o.f. & 1.7 & 1.7 & 1.7 & 1.7 \\
$e_{0}$ & -1.098 & -5.713 & -3.241 & -2.910 \\
$e_{1}$ & 2.739 & 2.192 & -2.698 & -2.691 \\
$e_{2}$ & -0.522 & -0.674 & 2.418 & 1.716 \\
$e_{3}$ & -2.248 & -3.301 & 1.270 & 1.762 \\
$e_{4}$ & 2.181 & 3.756 & -1.271 & -1.367 \\
$e_{5}$ & -0.196 & 0.441 & -0.288 & -1.134 \\
$e_{6}$ & 0.694 & -0.393 & -0.687 & -1.079 \\
$e_{7}$ & 7.546 & 4.361 & 3.883 & 0.815 \\
$e_{8}$ & 2.291 & 0.836 & 0.495 & -1.097 \\
$e_{9}$ & 4.052 & 1.040 & -0.070 & -1.316 \\
$e_{10}$ & 0.382 & 1.996 & -0.490 & -0.633 \\
$a_{2}(\mathrm{fm})$ & 0.636 & 0.641 & 0.646 & 0.650 \\
$a_{4}(\mathrm{fm})$ & 6.32 & 6.32 & 6.32 & 6.32 \\
\hline \hline
\end{tabular}




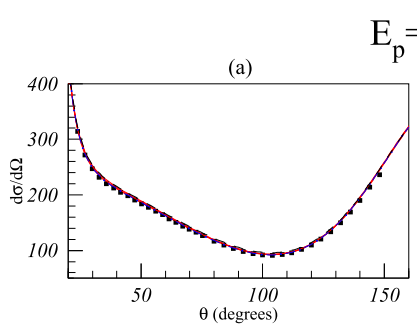

$$
\mathrm{E}_{\mathrm{p}}=3.0 \mathrm{MeV}
$$

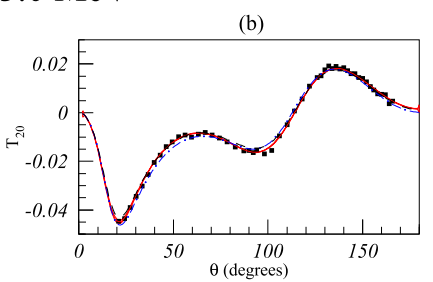

(c)

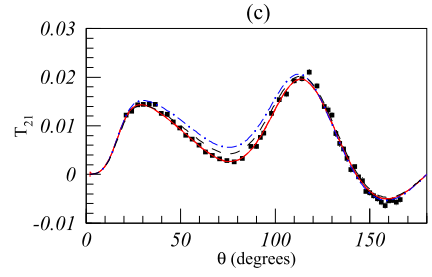

(e)
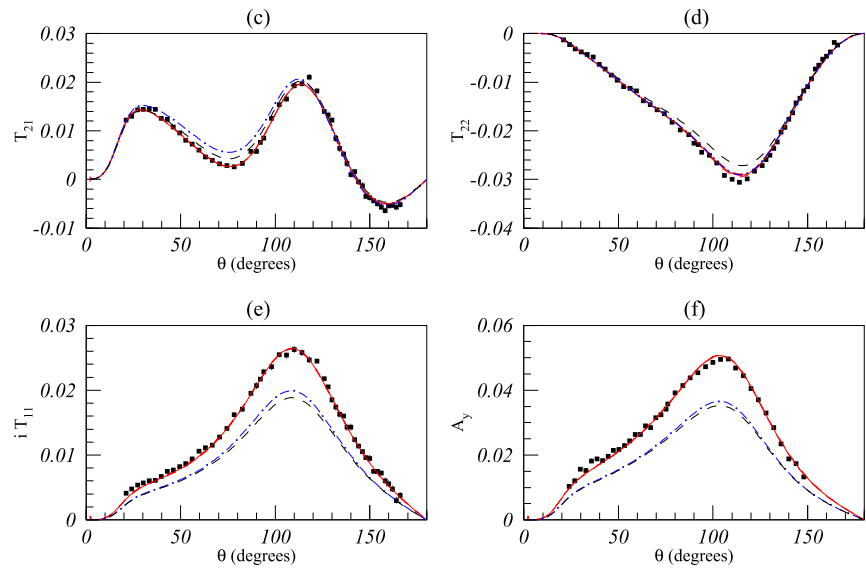

(f)

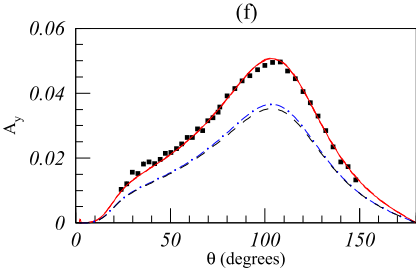

FIG. 2. Same as Fig. 1 but including all the $T=1 / 2$ subleading contact operators.

theoretical predictions using the subleading contact interaction determined with the complete fit at $E_{p}=3 \mathrm{MeV}$ are shown in Figs. 3-6, respectively. The predictions of the

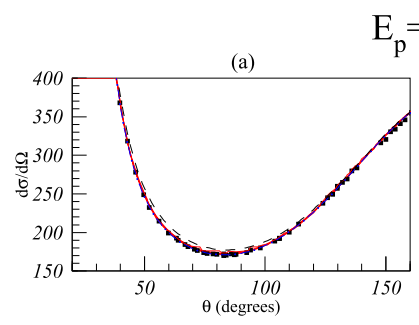

$$
\mathrm{E}_{\mathrm{p}}=1.0 \mathrm{MeV}
$$

(c)
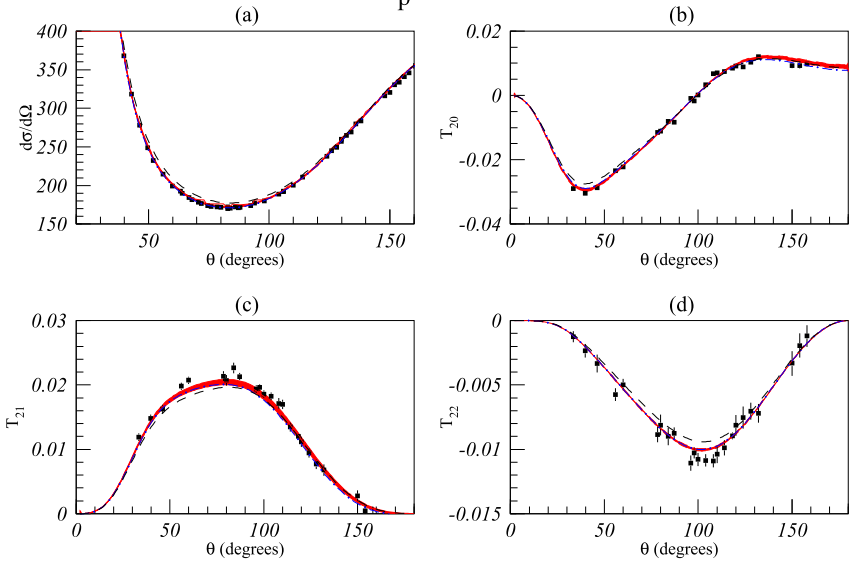

(d)
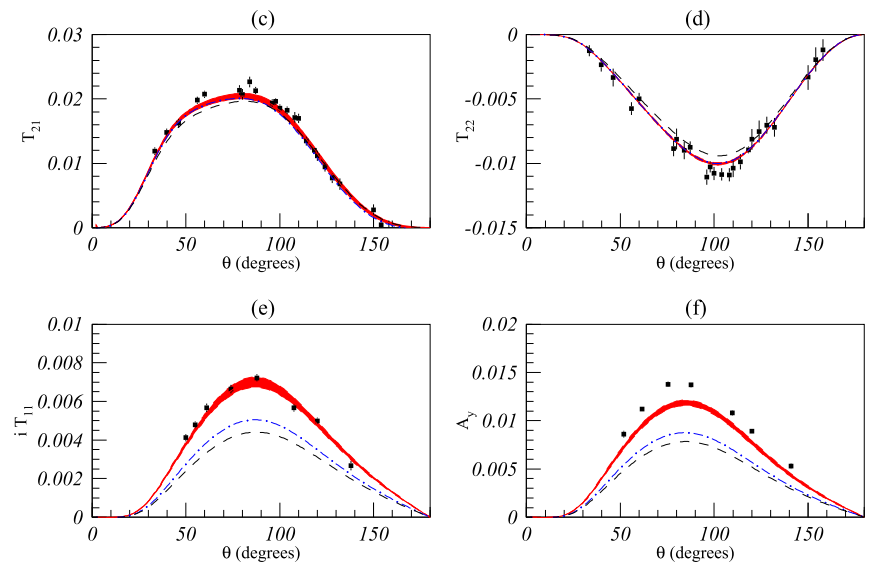

FIG. 3. Predictions of the models determined at $\Lambda$ between 200 and $500 \mathrm{MeV}$ (red bands) for a set of cross section and polarization $p$ - $d$ observables at $E_{p}=1 \mathrm{MeV}$ energy, as compared to the purely two-body AV18 interaction (dashed, black lines) and to the AV18+UIX two- and three-nucleon interaction (dashed-dotted, blue lines), in comparison to experimental data [44]. simplified models that include only the tensor and spin-orbit subleading contact operators show very similar behavior.

By inspection of the figures we observe an overall good agreement between theory and experiment for the observables, in particular the energy dependence of the analyzing power $A_{y}$ and the $i T_{11}$ observable is correctly encoded in the adopted contact interaction, although a small underprediction is still observed at the lowest energies. We have also investigated in Fig. 7 the $i T_{11}$ scattering observable at angle $\theta=88^{\circ}$, for which experimental data are available in the intermediate energy domain. From the figure we see that for this observable the simplified model including only the tensor and spin-orbit subleading operator is more accurate than the complete one, which is systematically below the data below $E_{p}=3 \mathrm{MeV}$, corresponding to center of mass energy $E_{\mathrm{cm}}=2 \mathrm{MeV}$. A simultaneous fit at different energies will presumably lead to a more accurate description in the very low-energy domain. The investigation of this possibility is left for future work.

\section{STUDY OF HIERARCHICAL STRUCTURES IN THE SUBLEADING TNI}

\section{A. Large- $N_{c}$ constraints}

It is interesting to explore whether the proposed model of TNI fulfills the hierarchy dictated by the large- $N_{c}$ limit of QCD, thus revealing a closer connection to the underlying theory of the strong interactions. Indeed, in the 't Hooft limit $[28,29]$ where the number of colors $N_{c} \rightarrow \infty$ and the strong coupling constant $g$ scales such as $g^{2} N_{c} \sim 1$, from the scaling of connected baryon-baryon amplitudes it is possible to derive the large- $N_{c}$ scaling of nuclear potentials $[46,47]$. Such scheme has proven to be qualitatively successful in the $N N$ case, providing in fact an expansion in $1 / N_{c}^{2}$, and has been applied to the $3 N$ case [48] as a guide to reduce the large number of operatorial structures. In particular, one finds that the scaling of the two-nucleon $\sigma \otimes \sigma$ and $\tau \otimes \boldsymbol{\tau}$ operators is twice suppressed with respect to $\mathbf{1} \otimes \mathbf{1}$ and $\boldsymbol{\sigma} \tau \otimes \boldsymbol{\sigma} \tau$, i.e.

$$
\boldsymbol{\sigma} \otimes \boldsymbol{\sigma} \sim \boldsymbol{\tau} \otimes \boldsymbol{\tau} \sim \frac{1}{N_{c}}, \quad \mathbf{1} \otimes \mathbf{1} \sim \boldsymbol{\sigma} \tau \otimes \boldsymbol{\sigma} \tau \sim N_{c} .
$$

This is in phenomenological agreement with the size of the two LECs, $C_{S}$ and $C_{T}$, corresponding to leading-order $N N$ contact interactions,

$$
\mathcal{L}_{N N}=-\frac{1}{2} C_{S}\left(N^{\dagger} N\right)^{2}-\frac{1}{2} C_{T}\left(N^{\dagger} \sigma N\right)^{2},
$$

where $\left|C_{S}\right| \gg\left|C_{T}\right|$. Notice that the most general leadingorder contact Lagrangian involving spin-isospin-1/2 baryons contains in principle four different operators,

$$
\begin{aligned}
\mathcal{L}_{N N} & =c_{1}\left(N^{\dagger} N\right)^{2}+c_{2}\left(N^{\dagger} \boldsymbol{\sigma} N\right)^{2}+c_{3}\left(N^{\dagger} \boldsymbol{\tau} N\right)^{2}+c_{4}\left(N^{\dagger} \boldsymbol{\sigma} \tau N\right)^{2} \\
& \equiv \sum_{i} c_{i} o_{i},
\end{aligned}
$$

which are related through Fierz-like identities by

$$
o_{3}=-o_{2}-2 o_{1}, \quad o_{4}=-3 o_{1} .
$$

These relations do not conform with the large- $N_{c}$ scaling (35). We observe, however, that the counting arguments, which lead 


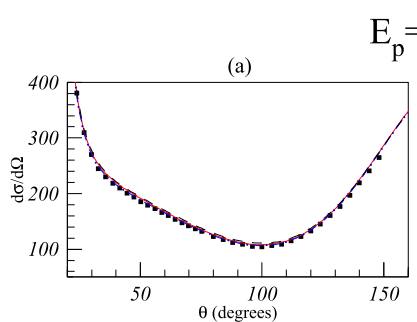

$\mathrm{E}_{\mathrm{p}}=2.5 \mathrm{MeV}$
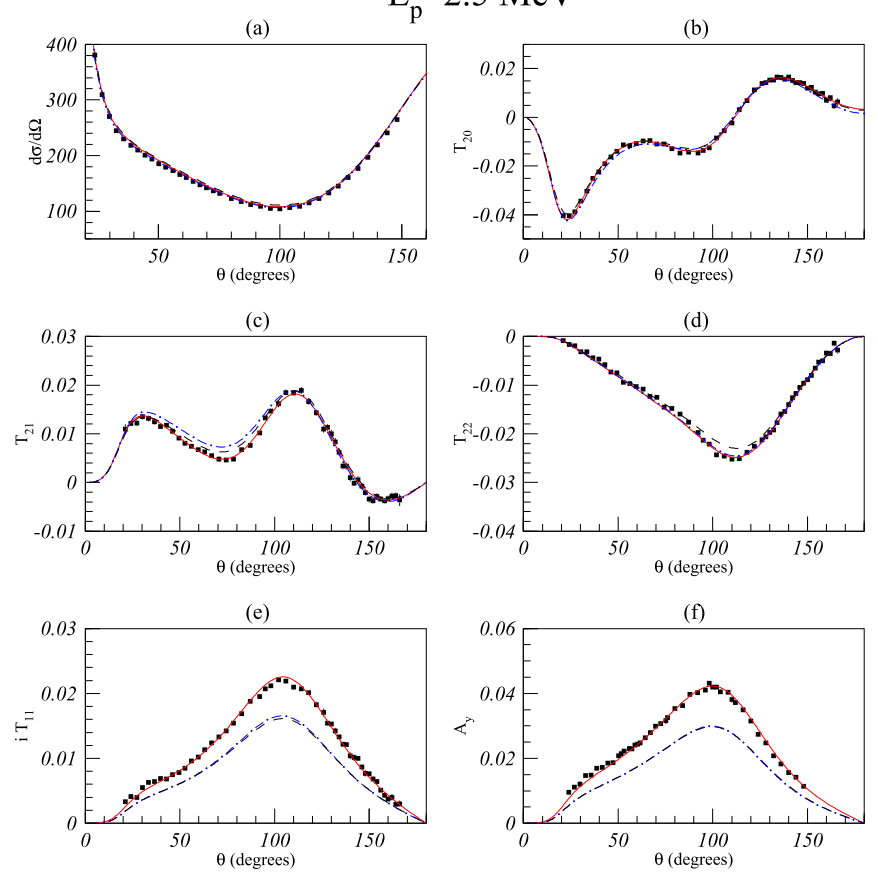

FIG. 4. Predictions of the model determined at $\Lambda=300 \mathrm{MeV}$ for a set of cross section and polarization $p$ - $d$ observables at $E_{p}=$ $2.5 \mathrm{MeV}$ proton energy (solid, red lines) as compared to the purely two-body AV18 interaction (dashed, black lines) and to the AV18+UIX two- and three-nucleon interaction (dashed-dotted, blue lines), in comparison to experimental data [27].

to the large- $N_{c}$ scaling never use the fact that the baryons are identical fermions. In particular, the same scaling would apply to scattering of distinguishable baryons. In this case we would have $c_{1} \sim c_{4} \sim O\left(N_{c}\right)$ while $c_{2} \sim c_{3} \sim O\left(1 / N_{c}\right)$. The indistinguishability of nucleons implies relations (38), which in turn allow us to cast the effective Lagrangian in the form (36) with

$$
C_{S}=-2\left(c_{1}-2 c_{3}-3 c_{4}\right), \quad C_{T}=-2\left(c_{2}-c_{3}\right),
$$

whence the conclusion on the relative size of $C_{S}$ and $C_{T}$. We thus learn that one way to implement the Pauli principle in the large- $N_{c}$ counting is to start with a redundant set of operators, establish the counting of the corresponding LECs, and impose the Pauli principle constraints afterwards.

In the notation of Table I of Ref. [23] there are 13 leading operators in the large- $N_{c}$ limit [48], so that the $3 N$ contact Lagrangian only depends on the 13 corresponding leading LECs,

$$
\begin{aligned}
\mathcal{L}_{3 N}= & c_{33} o_{33}+c_{37} o_{37}+c_{40} o_{40}+c_{43} o_{43}+c_{47} o_{47} \\
& +c_{51} o_{51}+c_{55} o_{55}+c_{59} o_{59}+c_{60} o_{60}+c_{61} o_{61} \\
& +c_{62} o_{62}+c_{63} o_{63}+c_{64} o_{64} .
\end{aligned}
$$

Using the Fierz identities obtained in Ref. [23], we can find their contributions to the ten LECs of the minimal basis,

$$
\begin{aligned}
& E_{1}=-\frac{1}{2} c_{33}-\frac{3}{2} c_{43}+\frac{3}{2} c_{55}, \\
& E_{2}=0,
\end{aligned}
$$

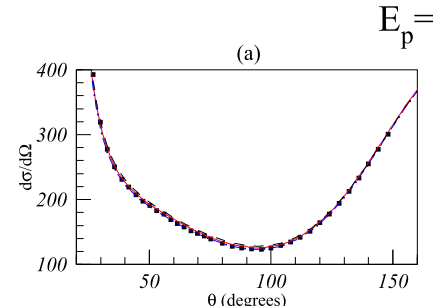

$\mathrm{E}_{\mathrm{p}}=2.0 \mathrm{MeV}$
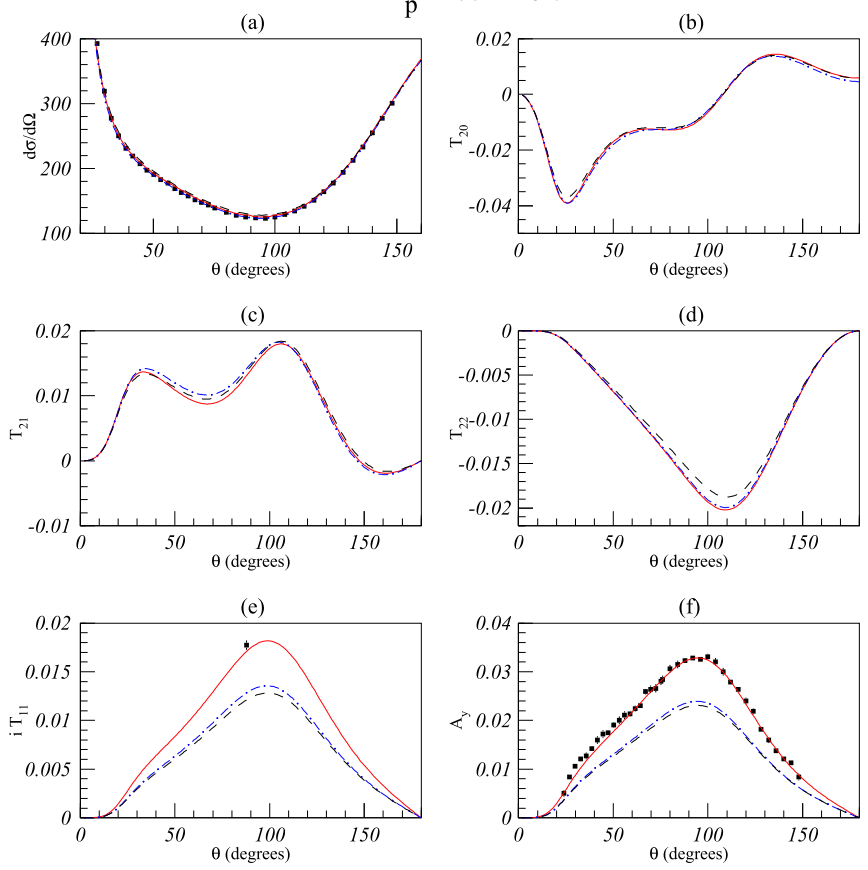

FIG. 5. Same as Fig. 4 but for $E_{p}=2 \mathrm{MeV}$ proton energy. Data are from Ref. [44].

$$
\begin{aligned}
E_{3}= & 0, \\
E_{4}= & -c_{43}-\frac{1}{3} c_{47}-\frac{1}{3} c_{51}-2 c_{59} \\
& -\frac{2}{3} c_{60}+\frac{2}{3} c_{61}-\frac{2}{3} c_{62},
\end{aligned}
$$

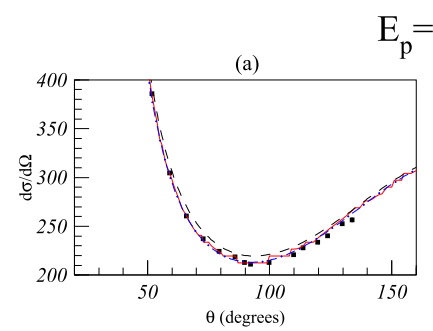

$\mathrm{E}_{\mathrm{p}}=0.65 \mathrm{MeV}$

(c)
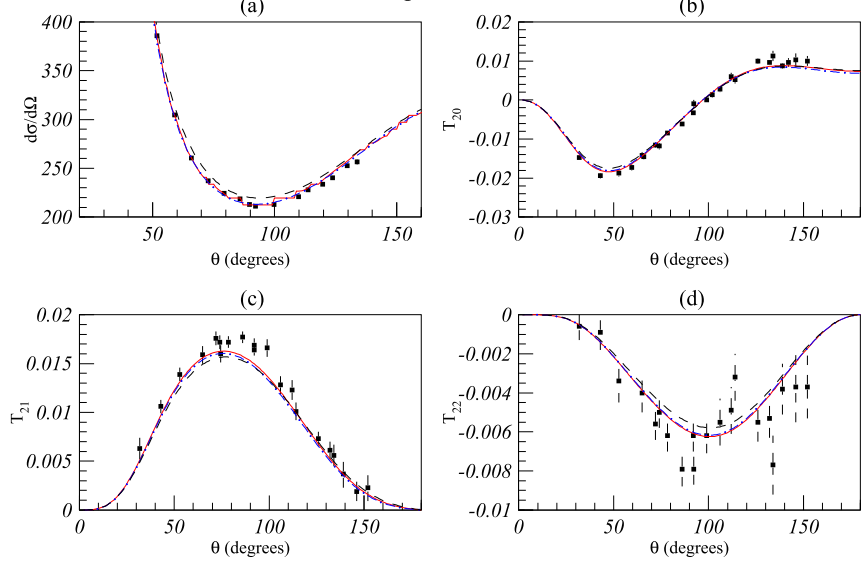

(d)
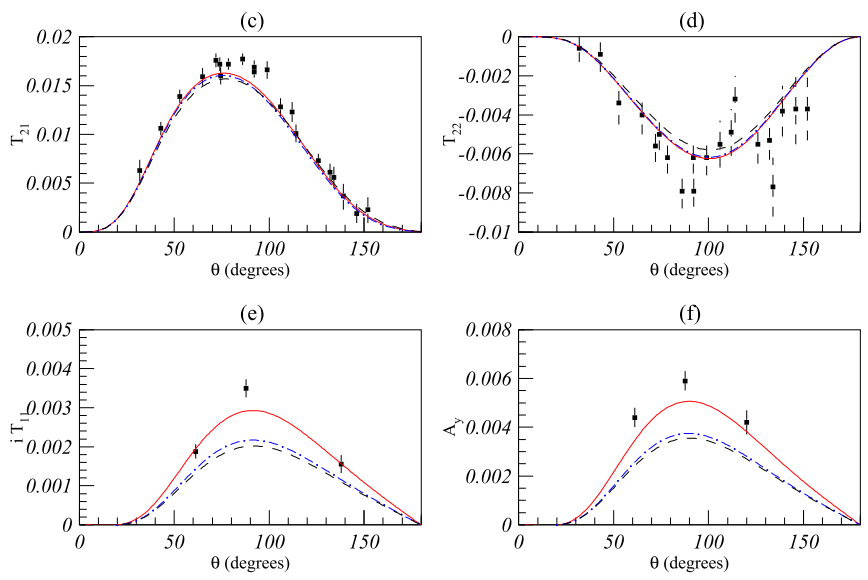

FIG. 6. Same as Fig. 4 but for proton energy $E_{p}=0.647 \mathrm{MeV}$. Data are from Ref. [45]. 


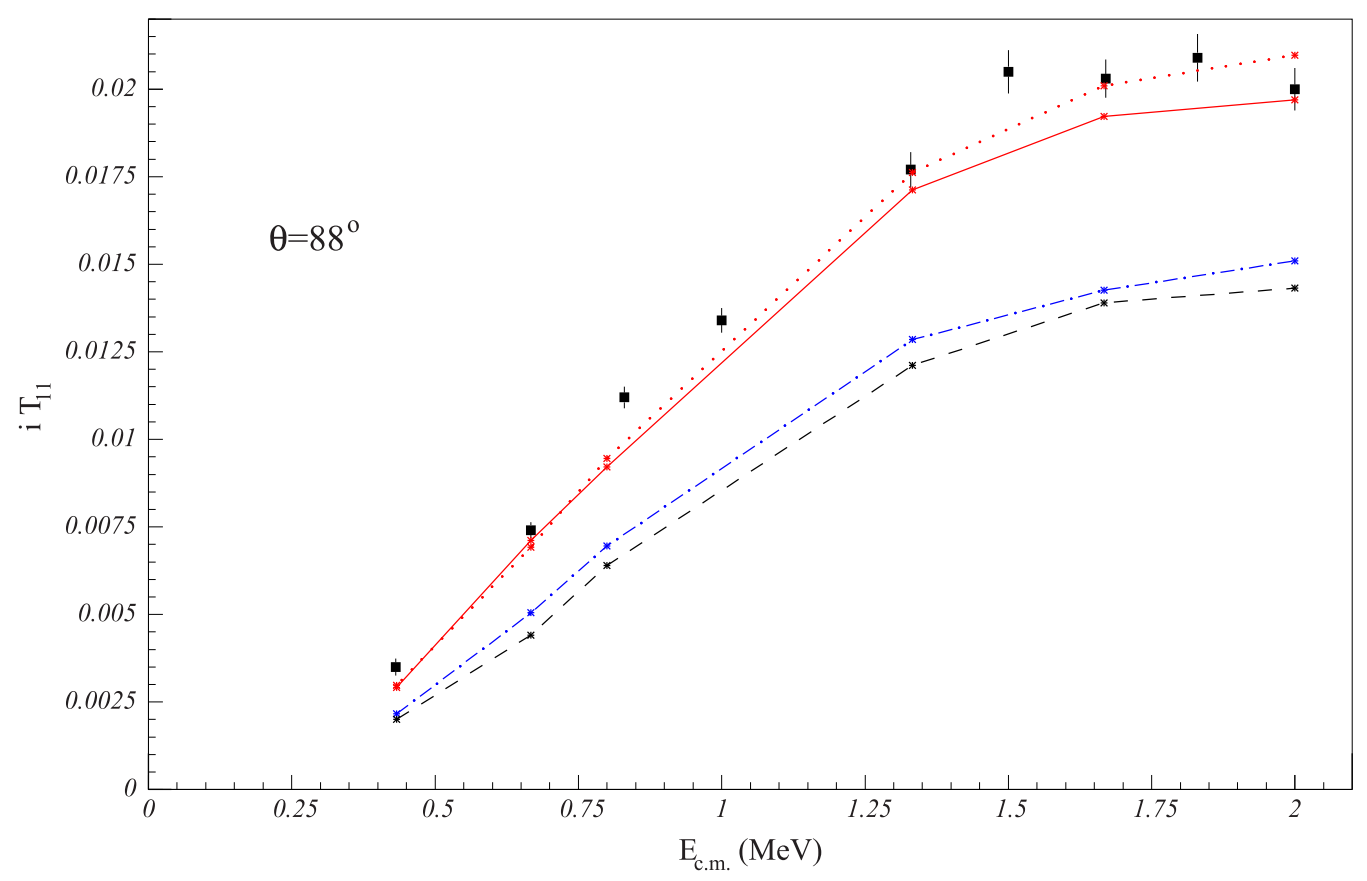

FIG. 7. Prediction of the model determined at $\Lambda=300 \mathrm{MeV}$ (red stars connected by solid lines) for the center-of-mass energy dependence of the vector polarization observable $i T_{11}$ at $\theta=88^{\circ}$ as compared to available experimental data of Ref. [45]. Also shown is the prediction of the simplified model corresponding to the second row of Table I (red stars, connected by dotted lines), of the purely two-body AV18 interaction (black stars connected by dashed lines) and to the AV18+UIX nuclear interaction (blues stars connected by dashed-dotted lines).

$$
\begin{aligned}
E_{5} & =0 \\
E_{6} & =-\frac{1}{3} c_{47}-\frac{1}{3} c_{51}-\frac{2}{3} c_{60}+\frac{2}{3} c_{61}+\frac{4}{3} c_{62} \\
E_{7} & =24 c_{40}-24 c_{51}+8 c_{63}+8 c_{64} \\
E_{8} & =8 c_{40}-8 c_{51}+4 c_{63}+4 c_{64} \\
E_{9} & =0 \\
E_{10} & =c_{37}+c_{40}-c_{47}-c_{51} .
\end{aligned}
$$

There are therefore only six independent combinations, so that the large- $N_{c}$ predictions can be summarized by the following constraints:

$$
E_{2}=E_{3}=E_{5}=E_{9}=0
$$

The projection onto the $T=1 / 2$ channel for this restricted interaction, along the same lines of Sec. II, leads to only five surviving LECs entering the $p$ - $d$ observables. As before, we can effectively impose the constraint

$$
2 E_{1}-E_{10}=0
$$

and understand that the LECs resulting from the fit are determined only up to an appropriate shift.

In order to test these large- $N_{c}$ predictions, we perform sixparameter fits to the same experimental data at $E_{p}=3 \mathrm{MeV}$, subjected to the large $-N_{c}$ and $T=1 / 2$ constraints, Eqs. (51) and (52), with the AV18 NN interaction plus the purely contact TNI of Eq (5). We ignore the Urbana IX potential in this case, since it also includes a short-distance component. For the sake of comparison, we show in Table III and Fig. 8 the fit results obtained ignoring the UIX interaction, which are of the same quality as the ones reported in Sec. III.

The results corresponding to the leading order of the large$N_{c}$ expansion are shown in Table IV and Fig. 9. By inspection of the table we can conclude that reasonable fits can be obtained in this limit, although at the cost of unnatural values for the spin-orbit LECs $E_{7}$. The increasing $\chi^{2} /$ d.o.f. for higher values of $\Lambda$ might be the consequence of the absence of longer-range components in the TNI from pion-exchange

TABLE III. Same as Table II but for the models that do not include the UIX TNI.

\begin{tabular}{lcccc}
\hline \hline$\Lambda(\mathrm{MeV})$ & 200 & 300 & 400 & 500 \\
\hline$\chi^{2} /$ d.o.f. & 1.6 & 1.7 & 1.7 & 1.7 \\
$e_{0}$ & 3.989 & -2.381 & -2.585 & -2.880 \\
$e_{1}$ & 2.149 & 2.791 & -3.250 & -2.850 \\
$e_{2}$ & -2.721 & -3.131 & 2.173 & 2.203 \\
$e_{3}$ & -1.829 & -3.490 & 0.601 & 1.224 \\
$e_{4}$ & 0.740 & 3.510 & -1.523 & -1.370 \\
$e_{5}$ & -0.535 & 0.007 & -0.452 & -0.665 \\
$e_{6}$ & 0.773 & -0.311 & -0.598 & -0.793 \\
$e_{7}$ & 7.265 & 3.490 & 1.750 & 2.291 \\
$e_{8}$ & 2.242 & 0.791 & -0.300 & -0.676 \\
$e_{9}$ & -0.244 & -1.690 & -1.183 & -0.859 \\
$e_{10}$ & -0.900 & 1.009 & -0.970 & -0.437 \\
$a_{2}(\mathrm{fm})$ & 0.639 & 0.645 & 0.647 & 0.645 \\
$a_{4}(\mathrm{fm})$ & 6.32 & 6.32 & 6.32 & 6.32 \\
\hline \hline
\end{tabular}




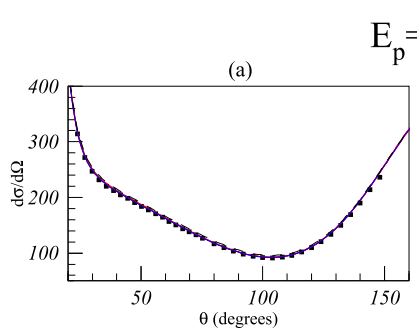

$\mathrm{E}_{\mathrm{p}}=3.0 \mathrm{MeV}$
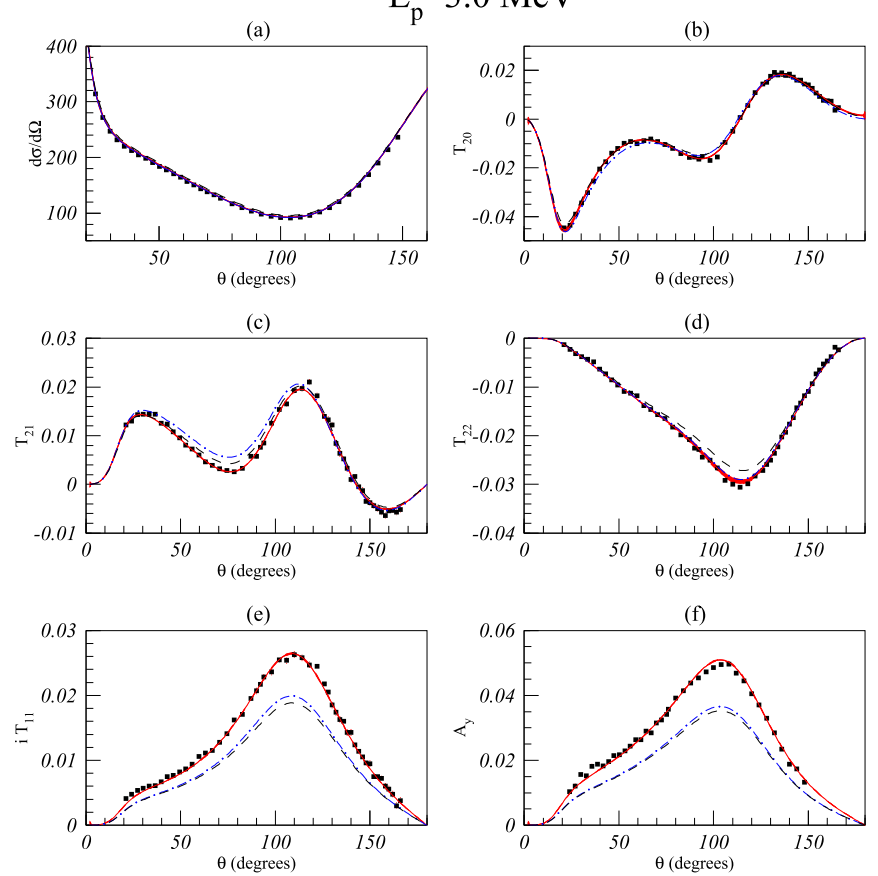

FIG. 8. Same as Fig. 1 but for the models that include all the $T=1 / 2$ subleading contact operators, without the UIX TNI.

contributions, which would also be leading in the large- $N_{c}$ counting.

\section{B. Relativistic counting}

A different kind of hierarchy among subleading contact operators can be deduced in the framework of the recently proposed relativistic counting for the $N N$ contact operators [30]. In this approach, one retains, in the leading-order Lagrangian, all relativistically invariant four-nucleon operators involving no space-time derivatives,

$$
\begin{aligned}
\mathcal{L}_{N N}^{(0)}= & \frac{1}{2}\left[C_{S}(\bar{\psi} \psi)(\bar{\psi} \psi)+C_{P}\left(\bar{\psi} \gamma_{5} \psi\right)\left(\bar{\psi} \gamma_{5} \psi\right)\right. \\
& +C_{V}\left(\bar{\psi} \gamma^{\mu} \psi\right)\left(\bar{\psi} \gamma_{\mu} \psi\right)+C_{A}\left(\bar{\psi} \gamma^{\mu} \gamma_{5} \psi\right)\left(\bar{\psi} \gamma_{\mu} \gamma_{5} \psi\right) \\
& \left.+C_{T}\left(\bar{\psi} \sigma^{\mu \nu} \psi\right)\left(\bar{\psi} \sigma_{\mu \nu} \psi\right)\right],
\end{aligned}
$$

TABLE IV. Same as Table II for the models corresponding to the leading order of the large- $N_{c}$ expansion.

\begin{tabular}{lcrrr}
\hline \hline$\Lambda(\mathrm{MeV})$ & 200 & 300 & \multicolumn{1}{c}{400} & \multicolumn{1}{c}{500} \\
\hline$\chi^{2} /$ d.o.f. & \multicolumn{1}{c}{2.0} & 1.9 & \multicolumn{1}{c}{2.0} & \multicolumn{1}{c}{2.1} \\
$e_{0}$ & -0.309 & -1.766 & -1.676 & -0.953 \\
$e_{1}$ & 0.328 & 0.400 & 0.557 & 0.717 \\
$e_{4}$ & -0.360 & 0.317 & 0.517 & 0.593 \\
$e_{6}$ & -0.060 & 0.115 & 0.162 & 0.297 \\
$e_{7}$ & 10.093 & 15.037 & 15.898 & 17.862 \\
$e_{8}$ & 3.559 & 5.371 & 5.397 & 5.961 \\
$e_{10}$ & 0.655 & 0.800 & 1.115 & 1.433 \\
$a_{2}(\mathrm{fm})$ & 0.668 & 0.653 & 0.640 & 0.628 \\
$a_{4}(\mathrm{fm})$ & 6.32 & 6.32 & 6.32 & 6.32 \\
\hline \hline
\end{tabular}

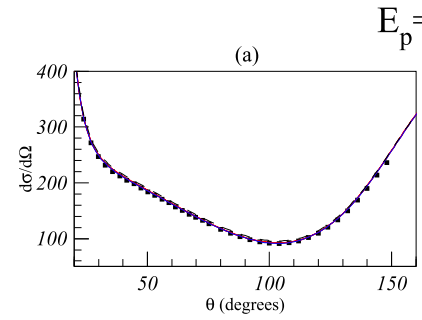

$\mathrm{E}_{\mathrm{p}}=3.0 \mathrm{MeV}$

(c)
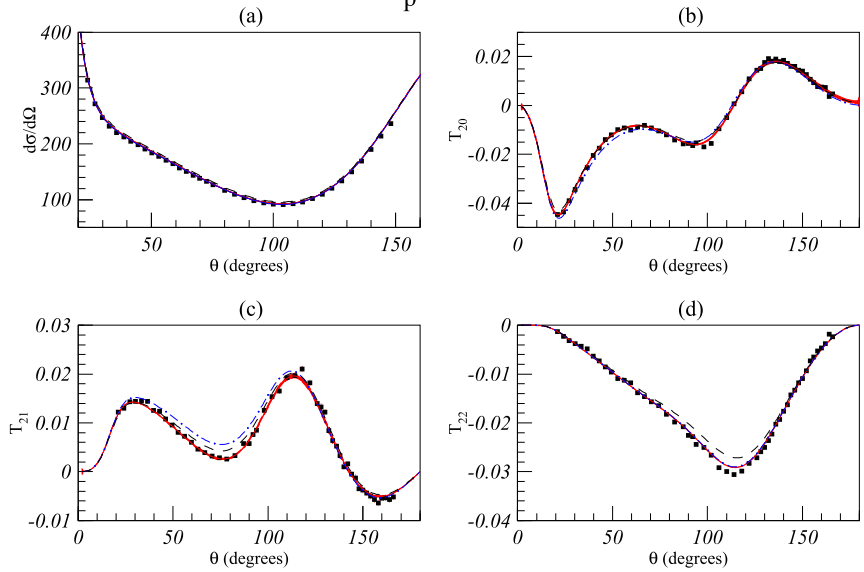

(d)
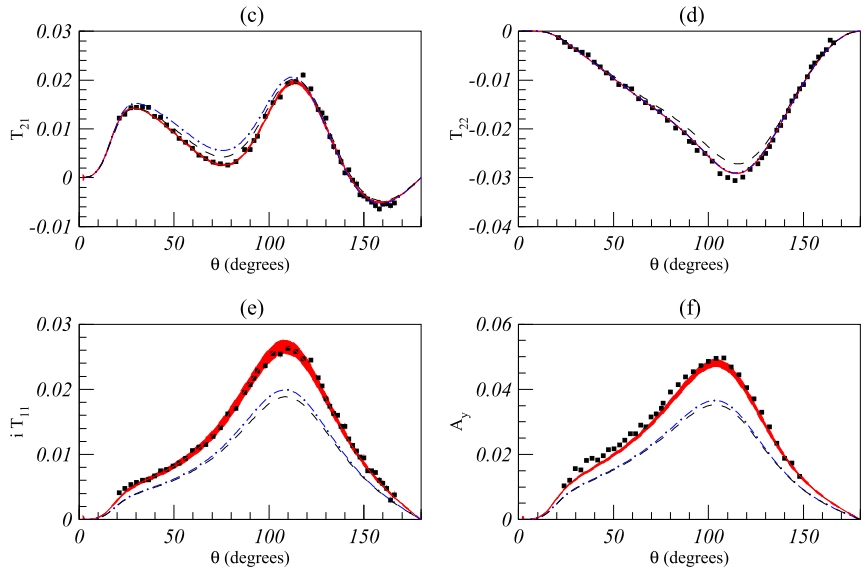

FIG. 9. Fit results in the leading order of the large- $N_{c}$ limit to a set of cross section and polarization $p$ - $d$ observables at $3 \mathrm{MeV}$ proton energy, for $\Lambda=200-500 \mathrm{MeV}$ (red bands) as compared to the purely two-body AV18 interaction (dashed, black lines) and to the AV18+UIX two- and three-nucleon interaction (dashed-dotted, blue lines).

where $\psi$ collects the Dirac spinor nucleon fields. In contrast to common practice, one does not expand around the static nucleon limit, which would amount to collapsing the five LECs onto two independent combinations, Eq. (36), which parametrize the central and spin-spin short-range potential. Instead, all five LECs are considered on an equal footing, generating further spin operators, among which the spin-orbit term. This procedure yields a much faster convergence of the low-energy expansion, since at each order there are more adjustable parameters. We can apply the same procedure to the three-nucleon case by writing all possible relativistically invariant six-nucleon operators, symmetric under isospin, charge-conjugation $(\mathcal{C})$, parity $(\mathcal{P})$, and time reversal $(\mathcal{T})$ transformations. The transformation properties of the different space-time and isospin structure inside fermion bilinears under the discrete symmetries are displayed in Table V. Based

TABLE V. Transformation properties of the fermion bilinears with the different elements of the Clifford and flavour algebra, and Levi-Civita tensor under parity $(\mathcal{P})$, charge conjugation $(\mathcal{C})$, and Hermitian conjugation (H.c.).

\begin{tabular}{lccccccc}
\hline \hline & 1 & $\gamma_{5}$ & $\gamma_{\mu}$ & $\gamma_{\mu} \gamma_{5}$ & $\sigma_{\mu \nu}$ & $\tau^{a}$ & $\epsilon_{\mu \nu \rho \sigma}$ \\
\hline $\mathcal{P}$ & + & - & + & - & + & + & - \\
$\mathcal{C}$ & + & + & - & + & - & $(-1)^{a+1}$ & + \\
H.c. & + & - & + & + & + & + & + \\
\hline \hline
\end{tabular}


TABLE VI. A complete, but nonminimal, set of Lorentz, isospin, $\mathcal{C}-, \mathcal{P}_{-}, \mathcal{T}$-invariant $3 N$ contact operators involving no space-time derivatives of fields.

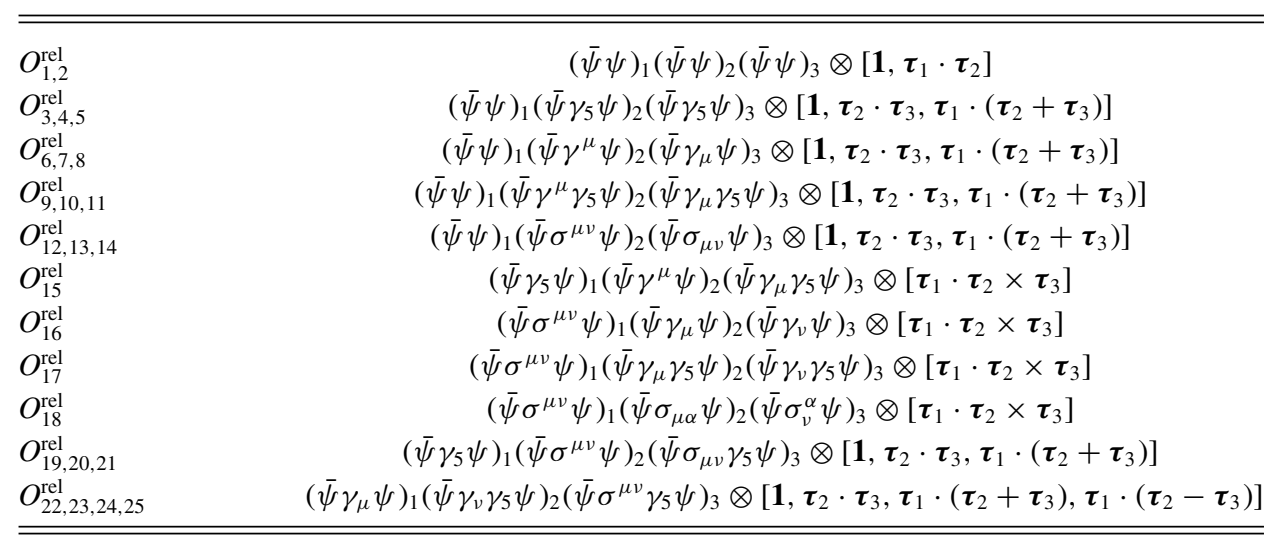

on these properties we can form a set of 25 different operators, displayed in Table VI.

Simultaneous rearrangements of Dirac and flavor indices between identical nucleon fields lead to Fierz identities, as detailed in the Appendix. As a result, the leading relativistic $3 N$ contact Lagrangian is written in terms of five independent operators,

$$
\begin{aligned}
\mathcal{L}_{3 N}^{(0)}= & -\left[E_{S}(\bar{\psi} \psi)(\bar{\psi} \psi)+E_{P}\left(\bar{\psi} \gamma_{5} \psi\right)\left(\bar{\psi} \gamma_{5} \psi\right)\right. \\
& +E_{V}\left(\bar{\psi} \gamma^{\mu} \psi\right)\left(\bar{\psi} \gamma_{\mu} \psi\right)+E_{A}\left(\bar{\psi} \gamma^{\mu} \gamma_{5} \psi\right)\left(\bar{\psi} \gamma_{\mu} \gamma_{5} \psi\right) \\
& \left.+E_{T}\left(\bar{\psi} \sigma^{\mu \nu} \psi\right)\left(\bar{\psi} \sigma_{\mu \nu} \psi\right)\right](\bar{\psi} \psi) \\
\equiv & -\sum_{X} E_{X} O_{X}, \quad X=S, P, V, A, T
\end{aligned}
$$

The nonrelativistic expansion of the nucleon fields,

$$
\psi(x)=\left(\begin{array}{c}
1+\frac{\nabla^{2}}{8 m^{2}} \\
-\frac{i}{2 m} \sigma \cdot \nabla
\end{array}\right) N(x)+O\left(Q^{3}\right) .
$$

allows to express the five operators $O_{X}$ in terms of the subleading operators defined in Ref. [23] as

$$
\begin{aligned}
O_{S}= & \left(N^{\dagger} N\right)^{3}+\frac{3}{8 m^{2}}\left[o_{127}-2 o_{75}\right] \\
O_{P}= & -\frac{1}{4 m^{2}} o_{36} \\
O_{V}= & \left(N^{\dagger} N\right)^{3}-\frac{1}{8 m^{2}}\left[4 o_{33}-4 o_{75}-4 o_{79}-2 o_{1}\right. \\
& \left.+2 o_{42}-2 o_{39}-o_{127}+2 o_{75}\right] \\
O_{A}= & \left(N^{\dagger} N\right)^{3}-\frac{1}{8 m^{2}}\left[2 o_{4}+2 o_{130}-2 o_{36}-2 o_{45}-2 o_{137}\right. \\
& \left.-2 o_{79}-2 o_{83}+o_{134}-2 o_{115}\right] \\
O_{T}= & -2\left(N^{\dagger} N\right)^{3}-\frac{1}{4 m^{2}}\left[2 o_{33}-2 o_{10}+2 o_{7}-4 o_{75}\right. \\
& +2 o_{42}+2 o_{53}-2 o_{36}-2 o_{45}-2 o_{137} \\
& \left.-2 o_{79}-2 o_{83}-o_{134}+2 o_{115}\right]
\end{aligned}
$$

where the relation

$$
\left(N^{\dagger} N\right)^{3}=-\left(N^{\dagger} \boldsymbol{\sigma} N\right) \cdot\left(N^{\dagger} \boldsymbol{\sigma} N\right)\left(N^{\dagger} N\right)
$$

has been used, also a consequence of Fierz identities. It is possible to express the above operators in the minimal basis [23], obtaining

$$
\begin{aligned}
O_{S}= & O_{0}+\frac{1}{m^{2}}\left[-\frac{3}{16} O_{1}-\frac{1}{8} O_{2}-\frac{1}{8} O_{3}-\frac{1}{6} O_{4}+\frac{1}{16} O_{5}\right. \\
& \left.+\frac{1}{48} O_{6}-\frac{5}{4} O_{7}-\frac{1}{4} O_{8}\right], \\
O_{P}= & -\frac{1}{4 m^{2}} O_{9}, \\
O_{V}= & O_{0}+\frac{1}{m^{2}}\left[\frac{3}{16} O_{1}-\frac{1}{8} O_{4}-\frac{3}{16} O_{5}-\frac{1}{16} O_{6}\right. \\
& \left.-\frac{1}{4} O_{7}-\frac{1}{4} O_{8}-\frac{1}{2} O_{9}-\frac{1}{4} O_{10}\right], \\
O_{A}= & O_{0}+\frac{1}{m^{2}}\left[\frac{3}{16} O_{1}-\frac{1}{4} O_{2}+\frac{1}{4} O_{3}-\frac{1}{24} O_{4}+\frac{1}{16} O_{5}\right. \\
& \left.+\frac{1}{48} O_{6}+\frac{11}{4} O_{7}+\frac{3}{4} O_{8}+\frac{1}{2} O_{9}+\frac{1}{4} O_{10}\right], \\
O_{T}= & -2 O_{0}+\frac{1}{m^{2}}\left[\frac{3}{8} O_{1}+\frac{3}{4} O_{2}-\frac{1}{4} O_{3}+\frac{1}{6} O_{4}-\frac{5}{8} O_{5}\right. \\
& \left.-\frac{5}{24} O_{6}-\frac{7}{2} O_{7}-\frac{3}{2} O_{8}-\frac{1}{2} O_{9}\right],
\end{aligned}
$$

where the operator $O_{0}$ contains the relativistic drift corrections [32],

$$
\begin{aligned}
O_{0}= & \left(N^{\dagger} N\right)^{3}+\frac{1}{16 m^{2}}\left[3\left(N^{\dagger} \overleftrightarrow{\nabla} N\right) \cdot\left(N^{\dagger} \overleftrightarrow{\nabla} N\right)\left(N^{\dagger} N\right)\right. \\
& -\left(N^{\dagger} \overleftrightarrow{\nabla} \tau^{a} N\right) \cdot\left(N^{\dagger} \overleftrightarrow{\nabla} \tau^{a} N\right)\left(N^{\dagger} N\right) \\
& -3\left(N^{\dagger} \overleftrightarrow{\nabla} \cdot \sigma N\right)\left(N^{\dagger} \overleftrightarrow{\nabla} \cdot \sigma N\right)\left(N^{\dagger} N\right) \\
& \left.-\left(N^{\dagger} \overleftrightarrow{\nabla} \cdot \sigma \tau^{a} N\right)\left(N^{\dagger} \overleftrightarrow{\nabla} \cdot \sigma \tau^{a} N\right)\left(N^{\dagger} N\right)\right] .
\end{aligned}
$$

Neglecting the latter we have

$$
\begin{aligned}
E_{0} & =E_{S}+E_{V}+E_{A}-2 E_{T}, \\
m^{2} E_{1} & =-\frac{3}{16} E_{S}+\frac{3}{16} E_{V}+\frac{3}{16} E_{A}+\frac{3}{8} E_{T} \\
& =-\frac{3}{16} E_{0}+\frac{3}{8} E_{V}+\frac{3}{8} E_{A},
\end{aligned}
$$




$$
\begin{aligned}
m^{2} E_{2} & =-\frac{1}{8} E_{S}-\frac{1}{4} E_{A}+\frac{3}{4} E_{T} \\
& =-\frac{1}{8} E_{0}+\frac{1}{8} E_{V}-\frac{1}{8} E_{A}+\frac{1}{2} E_{T}, \\
m^{2} E_{3} & =-\frac{1}{8} E_{S}+\frac{1}{4} E_{A}-\frac{1}{4} E_{T} \\
& =-\frac{1}{8} E_{0}+\frac{1}{8} E_{V}+\frac{3}{8} E_{A}-\frac{1}{2} E_{T}, \\
m^{2} E_{4} & =-\frac{1}{6} E_{S}-\frac{1}{8} E_{V}-\frac{1}{24} E_{A}+\frac{1}{6} E_{T} \\
& =-\frac{1}{6} E_{0}+\frac{1}{24} E_{V}+\frac{1}{8} E_{A}-\frac{1}{6} E_{T}, \\
m^{2} E_{5} & =\frac{1}{16} E_{S}-\frac{3}{16} E_{V}+\frac{1}{16} E_{A}-\frac{5}{8} E_{T} \\
& =\frac{1}{16} E_{0}-\frac{1}{4} E_{V}-\frac{1}{2} E_{T}, \\
m^{2} E_{6} & =\frac{1}{48} E_{S}-\frac{1}{16} E_{V}+\frac{1}{48} E_{A}-\frac{5}{24} E_{T} \\
& =\frac{1}{48} E_{0}-\frac{1}{12} E_{V}-\frac{1}{6} E_{T}, \\
m^{2} E_{7} & =-\frac{5}{4} E_{S}-\frac{1}{4} E_{V}+\frac{11}{4} E_{A}-\frac{7}{2} E_{T} \\
& =-\frac{5}{4} E_{0}+E_{V}+4 E_{A}-6 E_{T}, \\
m^{2} E_{8} & =-\frac{1}{4} E_{S}-\frac{1}{4} E_{V}+\frac{3}{4} E_{A}-\frac{3}{2} E_{T} \\
& =-\frac{1}{4} E_{0}+E_{A}-2 E_{T}, \\
m^{2} E_{9} & =-\frac{1}{4} E_{P}-\frac{1}{2} E_{V}+\frac{1}{2} E_{A}-\frac{1}{2} E_{T}, \\
m^{2} E_{10} & =-\frac{1}{4} E_{V}+\frac{1}{4} E_{A},
\end{aligned}
$$

with only four independent combinations of the subleading $3 N$ contact LECs. We notice in particular that the numerical coefficients entering in the expression of $E_{7}$ are larger by one order of magnitude compared to the other LECs. This might be at the origin of the phenomenological prominence of the spin-orbit interaction encoded in $E_{7}$, already proposed in Ref. [31].

In terms of the operators defined in Table VI the isospin projection reads

$$
\begin{aligned}
\left(O_{1}^{\text {rel }}\right)_{1 / 2} & =\frac{1}{2} O_{1}^{\text {rel }}-\frac{1}{2} O_{2}^{\text {rel }} \\
& =\frac{5}{4} O_{S}+\frac{1}{4} O_{P}+\frac{1}{4} O_{V}-\frac{1}{4} O_{A}+\frac{1}{8} O_{T}, \\
\left(O_{3}^{\text {rel }}\right)_{1 / 2} & =\frac{1}{2} O_{3}^{\text {rel }}-\frac{1}{6} O_{4}^{\text {rel }}-\frac{1}{6} O_{5}^{\text {rel }} \\
& =-\frac{1}{12} O_{S}+\frac{11}{12} O_{P}-\frac{1}{12} O_{V}+\frac{1}{12} O_{A}-\frac{1}{24} O_{T}, \\
\left(O_{6}^{\text {rel }}\right)_{1 / 2} & =\frac{1}{2} O_{6}^{\text {rel }}-\frac{1}{6} O_{7}^{\text {rel }}-\frac{1}{6} O_{8}^{\text {rel }} \\
& =-\frac{1}{3} O_{S}-\frac{1}{3} O_{P}+\frac{2}{3} O_{V}+\frac{1}{3} O_{A}-\frac{1}{6} O_{T}, \\
\left(O_{9}^{\text {rel }}\right)_{1 / 2} & =\frac{1}{2} O_{9}^{\text {rel }}-\frac{1}{6} O_{10}^{\text {rel }}-\frac{1}{6} O_{11}^{\text {rel }} \\
& =\frac{1}{3} O_{S}+\frac{1}{3} O_{P}+\frac{1}{3} O_{V}+\frac{2}{3} O_{A}+\frac{1}{6} O_{T}, \\
\left(O_{12}^{\text {rel }}\right)_{1 / 2} & =\frac{1}{2} O_{12}^{\text {rel }}-\frac{1}{6} O_{13}^{\text {rel }}-\frac{1}{6} O_{14}^{\text {rel }} \\
& =-O_{S}-O_{P}-O_{V}+O_{A}+\frac{1}{2} O_{T},
\end{aligned}
$$

whence one conclude that there is only one purely $T=3 / 2$ operator,

$$
O_{3 / 2}^{\mathrm{rel}}=O_{S}+O_{P}+O_{V}-O_{A}+\frac{1}{2} O_{T}
$$

and four purely $T=1 / 2$ combinations, e.g.,

$$
\left(O_{S}+3 O_{P}\right), \quad\left(O_{V}-4 O_{P}\right), \quad\left(O_{V}+O_{A}\right), \quad\left(3 O_{V}-O_{T}\right) .
$$

TABLE VII. Same of Table II for the model restricted at the leading order of the relativistic counting.

\begin{tabular}{lcccc}
\hline \hline$\Lambda(\mathrm{MeV})$ & 200 & 300 & 400 & 500 \\
\hline$\chi^{2} /$ d.o.f. & 2.2 & 2.2 & 2.3 & 2.1 \\
$e_{0}$ & -1.169 & -0.565 & -0.281 & 1.506 \\
$e_{1}$ & 0.202 & 0.245 & 0.377 & 1.077 \\
$e_{2}$ & 0.074 & 0.031 & 0.018 & -0.193 \\
$e_{3}$ & 0.067 & 0.139 & 0.240 & 0.857 \\
$e_{4}$ & 0.029 & 0.054 & 0.086 & 0.232 \\
$e_{5}$ & -0.135 & -0.106 & -0.138 & -0.219 \\
$e_{6}$ & -0.045 & -0.035 & -0.046 & -0.073 \\
$e_{7}$ & 0.533 & 1.343 & 2.373 & 8.850 \\
$e_{8}$ & -0.002 & 0.230 & 0.456 & 1.993 \\
$e_{9}$ & 0.974 & 0.606 & 1.451 & 4.552 \\
$e_{10}$ & -0.415 & -0.046 & -0.654 & -2.837 \\
$a_{2}(\mathrm{fm})$ & 0.707 & 0.619 & 0.583 & 0.646 \\
$a_{4}(\mathrm{fm})$ & 6.32 & 6.32 & 6.32 & 6.32 \\
\hline \hline
\end{tabular}

As before, in fitting to $p-d$ observables, one can impose the constraint

$$
3 E_{S}-E_{P}-4 E_{V}+4 E_{A}-12 E_{T}=0,
$$

with the understanding that the determination of the LECs is only valid up to a shift involving $h_{3 / 2}$.

In order to test the effectiveness of the relativistic counting, we also fit the constants $E_{X}, X=S, P, V, A, T$ subjected to the

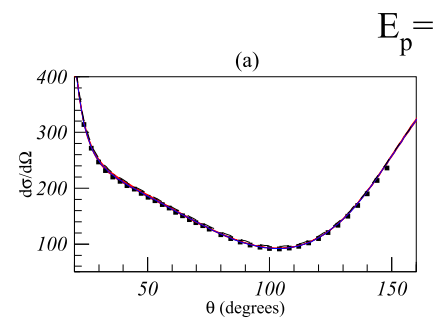

$$
\mathrm{E}_{\mathrm{p}}=3.0 \mathrm{MeV}
$$
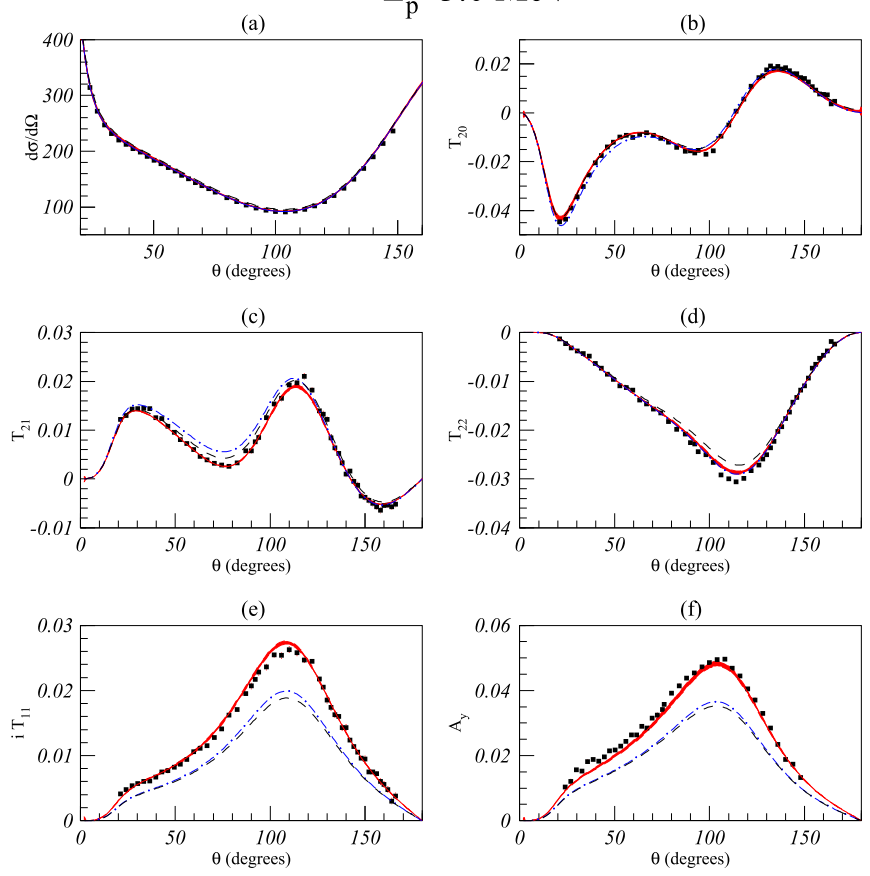

FIG. 10. Fit results in the leading order of the relativistic counting to a set of cross section and polarization $p$ - $d$ observables at $3 \mathrm{MeV}$ proton energy, for $\Lambda=200-500 \mathrm{MeV}$ (red bands) as compared to the purely two-body AV18 interaction (dashed, black lines) and to the AV18+UIX two- and three-nucleon interaction (dasheddotted, blue lines). 
$T=1 / 2$ constraint. As in the case of the large- $N_{c}$ limit we test this description in the framework of a purely pionless TNI. Specifically we consider a nuclear Hamiltonian consisting of the AV18 NN interaction and the leading and subleading $3 N$ contact terms implied by the Lagrangian (54). The latter can be expressed in the usual basis expressing the LECs $E_{i}$ 's in terms of the $E_{X}$ 's using the relations (68)-(78), with the $T=$ $1 / 2$ constraint expressed in Eq. (86). The fitted parameters are the adimensional $e_{0}, e_{V}, e_{A}$, and $e_{T}$, having defined the adimensional $e_{X}$ as

$$
E_{X}=\frac{e_{X}}{F_{\pi}^{4} \Lambda}, \quad X=V, A, T
$$

The results are displayed in Table VII. Compared to the unconstrained ten-parameter fit, the $\chi^{2} /$ d.o.f. is slightly increased, but a reasonable description is obtained for all adopted values of $\Lambda$. This may be considered as compatible with the leading-order character of the interaction, and gives support to the relativistic counting in the three-nucleon sector. We show in Fig. 10 the corresponding description of the $p-d$ scattering observables.

\section{CONCLUSIONS}

The aims of this paper are twofold. In the first part we discuss the possibility of determining the subleading contact three-body interaction from a fit procedure of selected binding energies and scattering data. In particular the fit includes the triton binding energy, the doublet and quartet $n$ - $d$ scattering lengths and several $p$ - $d$ scattering data. This being the first attempt to incorporate systematically scattering data in the determination of the TNI, we limit the fit of the $p$ - $d$ data to a single energy, $E_{p}=3 \mathrm{MeV}$, at which around 300 data points exist. However, we plan to extend the fit procedure to a simultaneous inclusion of data at several energies, below and above the deuteron breakup threshold. Regarding the results of the present analysis, they were satisfactory: the $\chi^{2} /$ d.o.f obtained after the fit was below 2, similar to the values obtained in the fit of the $N N$ potential in the two-nucleon sector. Moreover, they show a very small cutoff dependence. In this way we have shown that the subleading contact terms of the TNI provide enough flexibility to fit satisfactorily lowenergy elastic $N-d$ scattering observables, thus solving the longstanding discrepancy in some polarization observables. After decades of strong efforts to describe the $N N$ database with values of $\chi^{2} /$ d.o.f close to 1 , similar values were impossible to achieve in the three-nucleon sector even with the inclusion of very sophisticated TNI as the UIX type or from ChEFT up to N3LO. The angular-spin-isospin dependence of the TNI seems to be much more complicated than the forms used up to now. Here we have shown that this dependence can be opportunely collected in the sum of the ten terms given by the contact N4LO interaction.

To perform the fit the TNI contact interaction was summed to the AV18+UIX, widely used in the description of nuclear states. Interestingly, two-parameter fits including only the spin-orbit and tensor contact operators give already a good $\chi^{2} \sim 2$. Moreover, the resulting contact TNI, at least for the lower cutoffs, appears as a small correction as compared to the
UIX TNI. The unconstrained fit at $E_{p}=3 \mathrm{MeV}$ determines the ten combinations of LECs, $e_{i}, i=0, \ldots, 10$ (one LEC is fixed by the condition $h_{3 / 2}=0$ ) relevant to the $T=1 / 2$ channel, although with presumably large correlations among them. Stronger constraints could arise from a combined fit to observables at different energies. To evaluate the capability of the derived potential to describe other data, we explored the low-energy region, $E_{p}<3 \mathrm{MeV}$, in which several observables have been measured. We have observed an overall good agreement with a satisfactory description of the vector analyzing powers down to very low energies.

The relative importance of the ten subleading terms has been discussed in the second part of the paper, in which the whole TNI is of contact type. In fact, substantial improvement in the description of the same $p$ - $d$ observables is also provided by simplified versions of this interaction, given by the leading order of the recently proposed relativistic counting [30] or of the large- $N_{c}$ expansion [28,46-48]. Even though the naturality and the cutoff dependence of the involved LECs cannot be properly addressed, since we are using purely phenomenological models as the bulk of the $N N$ and $3 N$ interactions, the results suggest natural values of the LECs, with the marked exception of the spin-orbit ones, and a very mild cutoff dependence of the theoretical description in the range $\Lambda=200-500 \mathrm{MeV}$. It will be interesting to study this interaction in conjunction with chiral $N N$ and $3 N$ potentials or with purely contact nuclear interactions as implied in pionless EFT. Further investigation is also needed in order to test these models at higher energies and in larger systems. Studies along these lines are in progress.

\section{ACKNOWLEDGMENT}

The authors would like to thank Rocco Schiavilla for very useful discussions.

\section{APPENDIX: FIERZ-LIKE IDENTITIES}

In this Appendix we detail the Fierz-type relations used to reduce the relativistic operators in Sec. V B. The rearrangement of indices, indicated by round and square brackets for the two involved bilinears, concern Dirac indices, as, e.g.,

$$
\begin{aligned}
(\mathbf{1})[\mathbf{1}]= & \frac{1}{4}\left\{(\mathbf{1}][\mathbf{1})+\left(\gamma_{5}\right]\left[\gamma_{5}\right)+\left(\gamma^{\mu}\right]\left[\gamma_{\mu}\right)-\left(\gamma^{\mu} \gamma_{5}\right]\left[\gamma_{\mu} \gamma_{5}\right)\right. \\
& \left.+\frac{1}{2}\left(\sigma^{\mu \nu}\right]\left[\sigma_{\mu \nu}\right)\right\},
\end{aligned}
$$

and isospin indices, as

$$
(\mathbf{1})[\mathbf{1}]=\frac{1}{2}(\mathbf{1}][\mathbf{1})+\frac{1}{2}(\vec{\tau}] \cdot[\vec{\tau}) .
$$

Imposing the antisymmetry under exchange of identical fermion fields leads to relations (we omit in this Appendix the superscript "rel" for the sake of clarity) such as

$$
\begin{aligned}
O_{1}= & -\frac{1}{8}\left(O_{1}+O_{3}+O_{6}-O_{9}+\frac{1}{2} O_{12}\right. \\
& \left.+O_{2}+O_{4}+O_{7}-O_{10}+\frac{1}{2} O_{13}\right), \\
O_{2}= & -\frac{1}{8}\left[3\left(O_{1}+O_{3}+O_{6}-O_{9}+\frac{1}{2} O_{12}\right)\right. \\
& \left.-O_{2}-O_{4}-O_{7}+O_{10}-\frac{1}{2} O_{13}\right] .
\end{aligned}
$$


Similar relations can be obtained by using further identities, which can be obtained using the completeness of the Dirac bilinears,

$$
\begin{aligned}
& \text { (1) }\left[\gamma_{5}\right]=\frac{1}{4}\left\{\left(\gamma_{5}\right][\mathbf{1})+(\mathbf{1}]\left[\gamma_{5}\right)+\left(\gamma^{\mu} \gamma_{5}\right]\left[\gamma_{\mu}\right)-\left(\gamma^{\mu}\right]\left[\gamma_{\mu} \gamma_{5}\right)+2\left(\sigma^{\mu \nu}\right]\left[\sigma_{\mu \nu} \gamma_{5}\right)\right\} \text {, } \\
& \text { (1) }\left[\gamma^{\mu}\right]=\frac{1}{4}\left\{\left(\gamma^{\mu}\right][\mathbf{1})+(\mathbf{1}]\left[\gamma^{\mu}\right)-\left(\gamma^{\mu} \gamma_{5}\right]\left[\gamma_{5}\right)+\left(\gamma_{5}\right]\left[\gamma^{\mu} \gamma_{5}\right)-i\left(\gamma_{v}\right]\left[\sigma^{\mu \nu}\right)+i\left(\sigma^{\mu \nu}\right]\left[\gamma_{\nu}\right)\right. \\
& \left.+i\left(\gamma_{\nu} \gamma_{5}\right]\left[\sigma^{\mu v} \gamma_{5}\right)+i\left(\sigma^{\mu v} \gamma_{5}\right]\left[\gamma_{\nu} \gamma_{5}\right)\right\} \\
& \left(\gamma_{5}\right)\left[\gamma^{\mu} \gamma_{5}\right]=\frac{1}{4}\left\{\left(\gamma_{5}\right]\left[\gamma^{\mu} \gamma_{5}\right)+\left(\gamma^{\mu} \gamma_{5}\right]\left[\gamma_{5}\right)+(\mathbf{1}]\left[\gamma^{\mu}\right)-\left(\gamma^{\mu}\right][\mathbf{1})+i\left(\sigma^{\mu \nu}\right]\left[\gamma_{\nu}\right)+i\left(\gamma_{v}\right]\left[\sigma^{\mu \nu}\right)\right. \\
& \left.+i\left(\sigma^{\mu v} \gamma_{5}\right]\left[\gamma_{v} \gamma_{5}\right)-i\left(\gamma_{\nu} \gamma_{5}\right]\left[\sigma^{\mu v} \gamma_{5}\right)\right\} \\
& \text { (1) }\left[\gamma^{\mu} \gamma_{5}\right]=\frac{1}{4}\left\{(\mathbf{1}]\left[\gamma^{\mu} \gamma_{5}\right)+\left(\gamma^{\mu} \gamma_{5}\right][\mathbf{1})-\left(\gamma^{\mu}\right]\left[\gamma_{5}\right)+\left(\gamma_{5}\right]\left[\gamma^{\mu}\right)+i\left(\gamma_{\nu}\right]\left[\sigma^{\mu \nu} \gamma_{5}\right)\right. \\
& \left.+i\left(\sigma^{\mu v} \gamma_{5}\right]\left[\gamma_{\nu}\right)-i\left(\gamma_{\nu} \gamma_{5}\right]\left[\sigma^{\mu \nu}\right)+i\left(\sigma^{\mu \nu}\right]\left[\gamma_{\nu} \gamma_{5}\right)\right\} \\
& \text { (1) }\left[\sigma^{\mu \nu}\right]=\frac{1}{4}\left\{(\mathbf{1}]\left[\sigma^{\mu \nu}\right)+\left(\sigma^{\mu \nu}\right][\mathbf{1})+\left(\gamma_{5}\right]\left[\sigma^{\mu \nu} \gamma_{5}\right)+\left(\sigma^{\mu v} \gamma_{5}\right]\left[\gamma_{5}\right)+i\left(\sigma^{\mu \alpha}\right]\left[\sigma_{\alpha}^{\nu}\right)\right. \\
& \left.-\left(\sigma^{\nu \alpha}\right]\left[\sigma_{\alpha}^{\mu}\right)-\epsilon^{\mu \nu \alpha \beta}\left(\gamma_{\alpha}\right]\left[\gamma_{\beta} \gamma_{5}\right)+\epsilon^{\mu \nu \alpha \beta}\left(\gamma_{\alpha} \gamma_{5}\right]\left[\gamma_{\beta}\right)\right\} \text {, } \\
& \left(\gamma_{5}\right)\left[\sigma^{\mu v} \gamma_{5}\right]=\frac{1}{4}\left\{i\left(\gamma^{\mu}\right]\left[\gamma^{\nu}\right)-i\left(\gamma^{\nu}\right]\left[\gamma^{\mu}\right)-i\left(\gamma^{\mu} \gamma_{5}\right]\left[\gamma^{v} \gamma_{5}\right)+i\left(\gamma^{v} \gamma_{5}\right]\left[\gamma^{\mu} \gamma_{5}\right)+(\mathbf{1}]\left[\sigma^{\mu \nu}\right)\right. \\
& +\left(\sigma^{\mu \nu}\right][\mathbf{1})+\left(\gamma_{5}\right]\left[\sigma^{\mu \nu} \gamma_{5}\right)+\left(\sigma^{\mu \nu} \gamma_{5}\right]\left[\gamma_{5}\right)+i\left(\sigma^{\mu \alpha}\right]\left[\sigma_{\alpha}^{\nu}\right)-i\left(\sigma^{\nu \alpha}\right]\left[\sigma_{\alpha}^{\mu}\right) \\
& \left.+\epsilon^{\mu \nu \alpha \beta}\left(\gamma_{\alpha}\right]\left[\gamma_{\beta} \gamma_{5}\right)-\epsilon^{\mu \nu \alpha \beta}\left(\gamma_{\alpha} \gamma_{5}\right]\left[\gamma_{\beta}\right)\right\} \text {, } \\
& \left(\gamma_{5}\right)\left[\gamma^{\mu}\right]=\frac{1}{4}\left\{\left(\gamma_{5}\right]\left[\gamma^{\mu}\right)+\left(\gamma^{\mu}\right]\left[\gamma_{5}\right)+(\mathbf{1}]\left[\gamma^{\mu} \gamma_{5}\right)-\left(\gamma^{\mu} \gamma_{5}\right][\mathbf{1})-i\left(\gamma_{v}\right]\left[\sigma^{\mu \nu} \gamma_{5}\right)\right. \\
& \left.+i\left(\sigma^{\mu v} \gamma_{5}\right]\left[\gamma_{\nu}\right)+i\left(\gamma_{\nu} \gamma_{5}\right]\left[\sigma^{\mu \nu}\right)+i\left(\sigma^{\mu \nu}\right]\left[\gamma_{\nu} \gamma_{5}\right)\right\} \\
& \left(\sigma^{\mu \nu}\right)\left[\gamma_{\nu}\right]=\frac{1}{4}\left\{3 i\left[\left(\gamma^{\mu}\right][\mathbf{1})-(\mathbf{1}]\left[\gamma^{\mu}\right)-\left(\gamma^{\mu} \gamma_{5}\right]\left[\gamma_{5}\right)-\left(\gamma_{5}\right]\left[\gamma^{\mu} \gamma_{5}\right)\right]-\left(\sigma^{\mu \nu}\right]\left[\gamma_{\nu}\right)\right. \\
& \left.-\left(\gamma_{v}\right]\left[\sigma^{\mu v}\right)-\left(\sigma^{\mu v} \gamma_{5}\right]\left[\gamma_{\nu} \gamma_{5}\right)+\left(\gamma_{\nu} \gamma_{5}\right]\left[\sigma^{\mu v} \gamma_{5}\right)\right\} \text {, } \\
& \left(\sigma^{\mu \nu}\right)\left[\gamma_{\nu} \gamma_{5}\right]=\frac{1}{4}\left\{\left(\gamma_{\nu}\right]\left[\sigma^{\mu v} \gamma_{5}\right)-\left(\sigma^{\mu v} \gamma_{5}\right]\left[\gamma_{\nu}\right)-\left(\gamma_{\nu} \gamma_{5}\right]\left[\sigma^{\mu v}\right)-\left(\sigma^{\mu \nu}\right]\left[\gamma_{\nu} \gamma_{5}\right)\right. \\
& \left.+3 i\left[\left(\gamma^{\mu} \gamma_{5}\right][\mathbf{1})-(\mathbf{1}]\left[\gamma^{\mu} \gamma_{5}\right)-\left(\gamma^{\mu}\right]\left[\gamma_{5}\right)-\left(\gamma_{5}\right]\left[\gamma^{\mu}\right)\right]\right\}, \\
& \left(\gamma_{\mu}\right)\left[\sigma^{\mu \nu} \gamma_{5}\right]=\frac{1}{4}\left\{3 i\left[\left(\gamma^{\nu}\right]\left[\gamma_{5}\right)-\left(\gamma_{5}\right]\left[\gamma^{\nu}\right)+\left(\gamma^{v} \gamma_{5}\right][\mathbf{1})+(\mathbf{1}]\left[\gamma^{v} \gamma_{5}\right)\right]-\left(\gamma_{\mu}\right]\left[\sigma^{\mu \nu} \gamma_{5}\right)\right. \\
& \left.-\left(\sigma^{\mu \nu} \gamma_{5}\right]\left[\gamma_{\mu}\right)-\left(\gamma_{\mu} \gamma_{5}\right]\left[\sigma^{\mu \nu}\right)+\left(\sigma^{\mu \nu}\right]\left[\gamma_{\mu} \gamma_{5}\right)\right\} \text {, } \\
& \left(\sigma^{\mu \alpha}\right)\left[\sigma_{\alpha}^{\nu}\right]=\left(\sigma^{\nu \alpha}\right)\left[\sigma_{\alpha}{ }^{\mu}\right]+i\left(\sigma^{\mu \nu}\right][\mathbf{1})-i(\mathbf{1}]\left[\sigma^{\mu \nu}\right)+i\left(\sigma^{\mu \nu} \gamma_{5}\right]\left[\gamma_{5}\right)-i\left(\gamma_{5}\right]\left[\sigma^{\mu \nu} \gamma_{5}\right), \\
& \left(\gamma_{5}\right)\left[\sigma^{\mu \nu}\right]=\frac{1}{4}\left\{i\left(\gamma^{\mu} \gamma_{5}\right]\left[\gamma^{\nu}\right)-i\left(\gamma^{\nu} \gamma_{5}\right]\left[\gamma^{\mu}\right)-i\left(\gamma^{\mu}\right]\left[\gamma^{\nu} \gamma_{5}\right)+i\left(\gamma^{\nu}\right]\left[\gamma^{\mu} \gamma_{5}\right)+\left(\sigma^{\mu \nu}\right]\left[\gamma_{5}\right)\right. \\
& +\left(\gamma_{5}\right]\left[\sigma^{\mu \nu}\right)+\left(\sigma^{\mu \nu} \gamma_{5}\right][\mathbf{1})+(\mathbf{1}]\left[\sigma^{\mu \nu} \gamma_{5}\right)+i\left(\sigma^{\mu \alpha} \gamma_{5}\right]\left[\sigma_{\alpha}^{\nu}\right)-i\left(\sigma^{\nu \alpha} \gamma_{5}\right]\left[\sigma_{\alpha}^{\mu}\right) \\
& \left.-\epsilon^{\mu \nu \alpha \beta}\left(\gamma_{\alpha}\right]\left[\gamma_{\beta}\right)+\epsilon^{\mu \nu \alpha \beta}\left(\gamma_{\alpha} \gamma_{5}\right]\left[\gamma_{\beta} \gamma_{5}\right)\right\} \text {, } \\
& \left(\gamma^{\mu}\right)\left[\gamma^{v} \gamma_{5}\right]=\left(\gamma^{v}\right)\left[\gamma^{\mu} \gamma_{5}\right]+\frac{i}{2}\left\{\left(\sigma^{\mu v}\right]\left[\gamma_{5}\right)+\left(\gamma_{5}\right]\left[\sigma^{\mu v}\right)-\left(\sigma^{\mu v} \gamma_{5}\right][\mathbf{1})-(\mathbf{1}]\left[\sigma^{\mu v} \gamma_{5}\right)\right. \\
& \left.+\epsilon^{\mu \nu \alpha \beta}\left(\gamma_{\alpha}\right]\left[\gamma_{\beta}\right)+\epsilon^{\mu \nu \alpha \beta}\left(\gamma_{\alpha} \gamma_{5}\right]\left[\gamma_{\beta} \gamma_{5}\right)\right\} \text {. }
\end{aligned}
$$

Together with further isospin Fierz relations,

$$
\begin{aligned}
& (\vec{\tau})[\mathbf{1}]+(\mathbf{1})[\vec{\tau}]=(\vec{\tau}][\mathbf{1})+(\mathbf{1}][\vec{\tau}), \\
& (\vec{\tau})[\mathbf{1}]-(\mathbf{1})[\vec{\tau}]=-i(\vec{\tau}] \times[\vec{\tau}),
\end{aligned}
$$

one can establish the following linear relations,

$$
\begin{aligned}
& O_{3}=-\frac{1}{4}\left(O_{3}+\frac{1}{4} O_{19}+\frac{1}{2} O_{5}+\frac{1}{4} O_{20}\right) \\
& O_{4}=-\frac{1}{4}\left(O_{4}+\frac{1}{2} O_{5}-i O_{15}+\frac{1}{4} O_{21}\right) \\
& O_{5}=-\frac{1}{4}\left(3 O_{3}+O_{4}+i O_{15}+\frac{3}{4} O_{19}-\frac{1}{4} O_{20}+\frac{1}{4} O_{21}\right) \\
& O_{6}=-\frac{1}{4}\left(O_{6}+\frac{1}{2} O_{8}+i O_{22}+i O_{23}\right) \\
& O_{7}=-\frac{1}{4}\left(O_{7}+O_{8}-i O_{15}+O_{16}+i O_{24}\right)
\end{aligned}
$$




$$
\begin{aligned}
& O_{8}=-\frac{1}{4}\left(3 O_{6}+O_{7}+i O_{15}-O_{16}+3 i O_{22}-i O_{23}+i O_{24}\right), \\
& O_{9}=-\frac{1}{4}\left(O_{9}+\frac{1}{2} O_{11}-i O_{22}-\frac{i}{2} O_{24}+\frac{i}{2} O_{25}\right), \\
& O_{10}=-\frac{1}{4}\left(O_{10}+\frac{1}{2} O_{11}+i O_{15}+O_{17}-i O_{23}-\frac{i}{2} O_{24}-\frac{i}{2} O_{25}\right) \text {, } \\
& O_{11}=-\frac{1}{4}\left(3 O_{9}+O_{10}-i O_{15}-O_{17}-3 i O_{22}-i O_{23}-i O_{25}\right), \\
& O_{12}=-\frac{1}{4}\left(O_{12}+\frac{1}{2} O_{14}+O_{19}+\frac{1}{2} O_{21}+2 i O_{22}+i O_{24}+i O_{25}\right), \\
& O_{13}=-\frac{1}{4}\left(O_{13}+\frac{1}{2} O_{14}+O_{16}-O_{17}-O_{18}+O_{20}+\frac{1}{2} O_{21}+2 i O_{23}+i O_{24}-i O_{25}\right) \text {, } \\
& O_{14}=-\frac{1}{4}\left(3 O_{12}+O_{13}-O_{16}+O_{17}+O_{18}+3 O_{19}+O_{20}+6 i O_{22}+2 i O_{23}-2 i O_{25}\right), \\
& O_{15}=-\frac{1}{4}\left(i O_{11}-2 i O_{10}+2 O_{23}-O_{24}-O_{25}\right), \\
& O_{16}=-\frac{1}{4}\left(6 O_{7}-3 O_{8}-2 i O_{25}\right), \\
& O_{17}=-\frac{1}{4}\left(6 O_{10}-3 O_{11}-2 i O_{23}+i O_{24}+i O_{25}\right), \\
& O_{18}=O_{13}-\frac{1}{2} O_{14}+O_{20}-\frac{1}{2} O_{21} \\
& O_{19}=-\frac{1}{4}\left(O_{12}+\frac{1}{2} O_{14}+O_{19}+\frac{1}{2} O_{21}-2 i O_{22}-i O_{24}-i O_{25}\right), \\
& O_{20}=-\frac{1}{4}\left(O_{13}+\frac{1}{2} O_{14}-O_{16}+O_{17}-O_{18}+O_{20}+\frac{1}{2} O_{21}-2 i O_{23}-i O_{24}+i O_{25}\right) \text {, } \\
& O_{21}=-\frac{1}{4}\left(3 O_{12}+O_{13}+O_{16}-O_{17}+O_{18}+3 O_{19}+O_{20}-6 i O_{22}-2 i O_{23}+2 i O_{25}\right), \\
& O_{22}=\frac{i}{4}\left(O_{12}+\frac{1}{2} O_{14}-O_{19}-\frac{1}{2} O_{21}\right) \text {, } \\
& O_{23}=\frac{i}{4}\left(O_{13}+\frac{1}{2} O_{14}-O_{16}-O_{17}-O_{20}-\frac{1}{2} O_{21}\right), \\
& O_{24}=\frac{i}{4}\left(3 O_{12}+O_{13}+O_{16}+O_{17}-3 O_{19}-O_{20}\right), \\
& O_{25}=\frac{i}{4}\left(3 O_{12}-O_{13}-O_{14}-O_{16}-O_{17}-3 O_{19}+O_{20}+O_{21}\right), \\
& O_{15}=-\frac{i}{4}\left(2 O_{7}-O_{8}+2 i O_{25}\right) \\
& O_{22}=-\frac{1}{4}\left(3 i O_{9}+\frac{3}{2} i O_{11}-O_{22}-\frac{1}{2} O_{24}+\frac{1}{2} O_{25}\right), \\
& O_{23}=-\frac{1}{4}\left(3 i O_{10}+\frac{3}{2} i O_{11}+3 O_{15}+i O_{17}-O_{23}-\frac{1}{2} O_{24}-\frac{1}{2} O_{25}\right), \\
& O_{24}=-\frac{1}{4}\left(9 i O_{9}+3 i O_{10}-3 O_{15}-i O_{17}-3 O_{22}-O_{23}-O_{25}\right), \\
& O_{25}=-\frac{1}{4}\left(-9 i O_{9}+3 i O_{10}+3 i O_{11}-3 O_{15}-i O_{17}+3 O_{22}-O_{23}-O_{24}\right) \text {. }
\end{aligned}
$$

By examining the above relations one finds that all operators can be expressed as linear combinations of the $O_{X}, X=$ $S, P, V, A, T$, defined in Eq. (54). 
[1] D. R. Entem, R. Machleidt, and Y. Nosyk, Phys. Rev. C 96, 024004 (2017).

[2] E. Epelbaum, H. Krebs, and U.-G. Meißner, Eur. Phys. J. A 51, 53 (2015); Phys. Rev. Lett. 115, 122301 (2015).

[3] P. Reinert, H. Krebs, and E. Epelbaum, Eur. Phys. J. A 54, 86 (2018).

[4] M. Piarulli, L. Girlanda, R. Schiavilla, R. N. Pérez, J. E. Amaro, and E. R. Arriola, Phys. Rev. C 91, 024003 (2015).

[5] M. Piarulli et al., Phys. Rev. C 94, 054007 (2016).

[6] A. Ekström, G. Baardsen, C. Forssén, G. Hagen, M. HjorthJensen, G. R. Jansen, R. Machleidt, W. Nazarewicz, T. Papenbrock, J. Sarich, and S. M. Wild, Phys. Rev. Lett. 110, 192502 (2013).

[7] B. D. Carlsson et al., Phys. Rev. X 6, 011019 (2016).

[8] H. Krebs, A. Gasparyan, and E. Epelbaum, Phys. Rev. C 87, 054007 (2013); 85, 054006 (2012).

[9] E. Epelbaum, H. W. Hammer, and Ulf-G. Meißner, Rev. Mod. Phys. 81, 1773 (2009).

[10] R. Machleidt and D. R. Entem, Phys. Rept. 503, 1 (2011).

[11] E. Epelbaum, A. Nogga, W. Glöckle, H. Kamada, UlfG. Meißner, and H. Witala, Phys. Rev. C 66, 064001 (2002).

[12] V. Bernard, E. Epelbaum, H. Krebs, and Ulf-G. Meißner, Phys. Rev. C 77, 064004 (2008); 84, 054001 (2011).

[13] A. Kievsky, M. Viviani, and S. Rosati, Phys. Rev. C 64, 024002 (2001)

[14] L. E. Marcucci, A. Kievsky, L. Girlanda, S. Rosati, and M. Viviani, Phys. Rev. C 80, 034003 (2009)

[15] A. Kievsky, M. Viviani, and S. Rosati, Phys. Rev. C 52, R15 (1995)

[16] J. Golak et al., Eur. Phys. J. A 50, 177 (2014).

[17] M. Viviani, L. Girlanda, A. Kievsky, and L. E. Marcucci, Phys. Rev. Lett. 111, 172302 (2013).

[18] D. R. Entem, R. Machleidt, and H. Witała, Phys. Rev. C 65, 064005 (2002).

[19] A. Nogga, R. G. E. Timmermans, and U. van Kolck, Phys. Rev. C 72, 054006 (2005).

[20] M. C. Birse, Phys. Rev. C 74, 014003 (2006).

[21] M. P. Valderrama and D. R. Phillips, Phys. Rev. Lett. 114, 082502 (2015).
[22] A. Kievsky, M. Viviani, M. Gattobigio, and L. Girlanda, Phys. Rev. C 95, 024001 (2017).

[23] L. Girlanda, A. Kievsky, and M. Viviani, Phys. Rev. C 84, 014001 (2011).

[24] L. Girlanda, A. Kievsky, M. Viviani, and L. Marcucci, PoS CD 15, 103 (2016); EPJ Web Conf. 113, 04009 (2016).

[25] R. B. Wiringa, V. G. J. Stoks, and R. Schiavilla, Phys. Rev. C 51, 38 (1995).

[26] B. S. Pudliner, V. R. Pandharipande, J. Carlson, S. C. Pieper, and R. B. Wiringa, Phys. Rev. C 56, 1720 (1997).

[27] S. Shimizu et al., Phys. Rev. C 52, 1193 (1995).

[28] G. 't Hooft, Nucl. Phys. B 72, 461 (1974).

[29] E. Witten, Nucl. Phys. B 160, 57 (1979).

[30] X. L. Ren, K. W. Li, L. S. Geng, B. W. Long, P. Ring, and J. Meng, Chin. Phys. C 42, 014103 (2018).

[31] A. Kievsky, Phys. Rev. C 60, 034001 (1999).

[32] L. Girlanda, S. Pastore, R. Schiavilla, and M. Viviani, Phys. Rev. C 81, 034005 (2010).

[33] A. Kievsky, S. Rosati, M. Viviani, L. E. Marcucci, and L. Girlanda, J. Phys. G 35, 063101 (2008).

[34] A. Kievsky, Nucl. Phys. A 624, 125 (1997).

[35] A. Manohar and H. Georgi, Nucl. Phys. B 234, 189 (1984).

[36] H. Georgi, Phys. Lett. B 298, 187 (1993).

[37] K. Schoen et al., Phys. Rev. C 67, 044005 (2003).

[38] W. Dilg, L. Koester, and W. Nistler, Phys. Lett. B 36, 208 (1971).

[39] W. Glöckle, H. Witała, D. Hüber, H. Kamada, and J. Golak, Phys. Rep. 274, 107 (1996).

[40] A. Kievsky et al., Phys. Rev. C 63, 024005 (2001).

[41] T. Munson, J. Sarich, S. Wild, S. Benson, and L. McInnes, TAO 2.0 Users Manual, Technical Report ANL/MCS-TM-322, http://www.mcs.anl.gov/tao

[42] A. Kievsky, S. Rosati, W. Tornow, and M. Viviani, Nucl. Phys. A 607, 402 (1996).

[43] S. Ishikawa, Phys. Rev. C 75, 061002 (2007).

[44] M. H. Wood et al., Phys. Rev. C 65, 034002 (2002).

[45] C. R. Brune et al., Phys. Rev. C 63, 044013 (2001).

[46] D. B. Kaplan and A. V. Manohar, Phys. Rev. C 56, 76 (1997).

[47] D. B. Kaplan and M. J. Savage, Phys. Lett. B 365, 244 (1996).

[48] D. R. Phillips and C. Schat, Phys. Rev. C 88, 034002 (2013). 